\title{
On a Third-Order Phase Transition
}

\author{
Theodor Eisele
}

Institut für Angewandte Mathematik, Universität Heidelberg, Im Neuenheimer Feld 294, D-6900 Heidelberg, Federal Republic of Germany

Abstract. The asymptotic behaviour of random variables of the general form

$$
\ln \sum_{i=1}^{\kappa^{N}} \exp \left(N^{1 / p} \beta \zeta_{i}\right)
$$

with independent identically distributed random variables $\zeta_{i}$ is studied. This generalizes the random energy model of Derrida. In the limit $N \rightarrow \infty$, there occurs a particular kind of phase transition, which does not incorporate a bifurcation phenomenon or symmetry breaking. The hypergeometric character of the problem (see definitions of Sect. 4), its $\Phi$-function, and its entropy function are discussed.

\section{Introduction}

The great majority of solvable mathematical models of mean field type, which show phase transitions, are closely related to bifurcation problems in the order parameters. For example, many magnetic spin models are of this type. The general features of these models may be roughly summarized in the following way: The calculation of the free energy in the thermodynamic limit is equivalent to a large deviation problem, which by Laplace's method goes over to a variational principle for the free energy. At an extremum, the first derivative with respect to the order parameters must necessarily vanish. In most interesting, exactly soluble examples this condition implies a bifurcation phenomenon.

In this paper, we study a phase transition phenomenon of a completely different type. In fact, we shall see below that we have to do with a kind of iterated large deviation problem. It is this iteration which provokes the phase transition. On the other hand, the models are simple enough so that no additional bifurcation phenomena appear. Since the mean free energy is once, but not twice, continuously differentiable at the critical point, we may speak of a third-order phase transition.

We now describe the models in more detail. Let us consider a probability measure $\varrho$ on $\mathbb{R}$, which has an exponentially decreasing tail distribution at $+\infty$. 
This means roughly that

$$
\varrho[x,+\infty) \approx \exp \left(-b x^{p /(p-1)}\right) \text { for } x \rightarrow \infty
$$

for some $p \in[1, \infty)$ and $b>0$. (See Sect. 2 for the precise definition.) Take a sequence $\left(\zeta_{i}\right)$ of independent random variables with the common distribution $\varrho$, and define for $\beta \geqq 0, \kappa \in\{2,3, \ldots\}$

$$
Z_{N}=Z_{N}(\beta, \varrho, \kappa)=\sum_{i=1}^{\kappa^{N}} \exp \left\{N^{1 / p} \beta \zeta_{i}-N\left(\ln \kappa+\gamma \beta^{p}\right)\right\} .
$$

The term $\ln \kappa+\gamma \beta^{p}$ is introduced only for normalization. We are interested in the limit of $N^{-1} \ln Z_{N}$.

The main result of this paper is the stochastic convergence of $N^{-1} \ln Z_{N}$ to a deterministic function $\psi(\beta, \varrho, \ln \kappa)$ which exhibits the following non-analytic behaviour in $\beta$ : the function $\beta \mapsto \psi(\beta, \varrho, \ln \kappa)$ is once but not twice continuously differentiable. More precisely, there exists $\beta_{C}=\beta_{C}(\varrho, \ln \kappa)$ such that

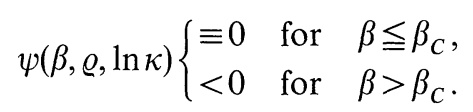

Moreover,

$$
\lim _{\beta \backslash \beta_{C}} \frac{d}{d \beta} \psi=0, \text { but } \lim _{\beta \backslash \beta_{C}} \frac{d^{2}}{d \beta^{2}} \psi<0 .
$$

In this sense, we say that the random variables $\ln Z_{N}$ give rise to a phase transition of third order.

This problem has first been studied by Derrida [2] for the case of a Gaussian distribution

$$
\varrho(d x)=(2 \pi)^{-1 / 2} \exp \left(-\frac{x^{2}}{2}\right) d x,
$$

i.e. $b=\frac{1}{2}, p=2$, and $\kappa=2$. He showed by direct calculations that

$$
\lim _{N \rightarrow \infty} N^{-1} E \ln Z_{N}=\left\{\begin{array}{lll}
0 & \text { for } & \beta \leqq \beta_{C}=\sqrt{2 \ln 2}, \\
-\beta^{2} / 2+\beta \cdot \beta_{C}-\ln 2 & \text { for } & \beta>\beta_{C} .
\end{array}\right.
$$

Derrida regarded the $\zeta_{i}$ as independent energy levels and called the system the random energy model. The expression (1.6) then corresponds to the mean free energy in the thermodynamic limit. The random energy model has been introduced in order to get a better understanding of the Sherrington-Kirkpatrick (SK) model for spin glasses [8]. In spite of the many papers devoted to solving the SK model, there is still no satisfactory theory for its solution. However, it is not difficult to check that an iterated large deviation problem is also involved in the SK model. One of the methods applied there was the replica trick, which however gave incorrect results. (See [6] for a detailed discussion of the replica method.)

Though we shall not comment on the Sherrington-Kirkpatrick model in this paper, we explain the replica method for our models in Sect. 6, and we shall see that this method would also fail here. Moreover, we will point out that the behaviour of $N^{-1} \ln Z_{N}$ and the results obtained by the replica method contain 
different information about the $\Phi$-function, namely its left and right derivatives at zero, which are identical only in cases without phase transition. At the end of Sect. 6, we shall propose a new method, which in our case gives the correct answer.

The generalization with respect to Derrida's result is various. First, we admit a general probability measure $\varrho$ instead of the standard normal distribution. Second, we treat the problem by the methods of the theory of large deviations, instead of the special and complicated analytical calculations in [2]. In doing so, we get a better insight into what really happens mathematically. Third, we show not only that the mean $N^{-1} E \ln Z_{N}$ converges, but the random variables themselves. Finally, we discuss the $\Phi$-function and the entropy function and explain the hypergeometric character of the problem.

In fact, if we write the $\Phi$-function $\Phi_{N}$ of $\ln Z_{N}$ (see [6]) in the form

$$
\begin{aligned}
\Phi_{N}(t) & =N^{-1} \ln E \exp \left\{t \ln Z_{N}\right\} \\
& =N^{-1} \ln E \exp \left[N t N^{-1} \ln \kappa^{-N} \sum_{i=1}^{\kappa^{N}} \exp \left\{N\left(N^{(1-p) / p} \beta \zeta_{i}-\gamma \beta^{p}\right)\right\}\right],
\end{aligned}
$$

it becomes obvious that we have to do with an iterated application of the "large deviation operator" $N^{-1} \ln E \exp N$. Recall that informally Laplace's method means

$$
\lim _{N \rightarrow \infty} N^{-1} \ln E \exp N(\cdot) \approx \operatorname{ess} \sup (\cdot) .
$$

By the form of (1.7), it is thus not surprising that, in some region, the asymptotic behaviour of $N^{-1} \ln Z_{N}$ is described by a hypergeometric entropy function, i.e.

$$
\operatorname{Prob}\left\{N^{-1} \ln Z_{N} \in d x\right\} \approx \exp (-\exp (N j(x))
$$

for some function $j$ and some $x \in \mathbb{R}$. Consequently, we shall also find a (one-sided) hypergeometric convergence of $N^{-1} \ln Z_{N}$. Unfortunately however, we were not able to calculate the asymptotic $\Phi$-function

$$
\Phi(t)=\lim _{N} \Phi_{N}(t) \text { for all } t .
$$

Here, we are left with some open questions.

In Sect. 2 , the fundamental assumption (1.1) on the measure $\varrho$ is made precise and presented in several equivalent versions. The limit of the means $N^{-1} E \ln Z_{N}(\beta, \varrho, \kappa)$ and the occurrence of the third-order phase transition are stated in Theorem 3.1. In Sect. 4, we discuss the hypergeometric character of the problem in the region $(-\infty, \psi(\beta, \varrho, \ln \kappa))$. We show then in Sect. 5 the different types of convergence of the random variables $N^{-1} \ln Z_{N}$, depending on whether $\beta<\beta_{C}$ or not. Finally, the $\Phi$-function and the entropy function are discussed in Sect. 6. The mathematical reason for the appearance of a phase transition ("sticking of the maximal point") is explained in Sect. 7, which gives the necessary preparations for the proofs of the results. The proofs themselves are then described in the rest of the paper. 


\section{The Condition on the Measure $\varrho$}

In this section we investigate the condition on $\varrho$ which we shall need later. So let $\varrho$ be a probability measure on $\mathbb{R}$ which satisfies

(i) $\int \exp (t x) \varrho(d x)<+\infty$ for all $t \in \mathbb{R}$,

$$
\left\{\begin{array}{l}
\text { (ii) there exists } p \in[1, \infty) \text { and } \gamma \in(0, \infty) \text { such that } \\
\lim _{t \rightarrow \infty} t^{-p} \ln \int \exp (t x) \varrho(d x)=\gamma \text {. }
\end{array}\right.
$$

Define the transformed measure $\varrho_{t}$ on $\mathbb{R}$ by

$$
\varrho_{t}(d x)=\exp (t x) \varrho(d x) / \int \exp \left(t x^{\prime}\right) \varrho\left(d x^{\prime}\right) .
$$

By l'Hospital's rule, (2.2) is equivalent to

$$
\lim _{t \rightarrow \infty} t^{-(p-1)} \int x \varrho_{t}(d x)=\gamma \cdot p .
$$

We start to give several examples of measures satisfying condition (2.2) using the following result:

Proposition 2.1. The probability measure $\varrho$ on $\mathbb{R}$ satisfies (2.2) with $p=1$ if and only if its restriction to $(0, \infty)$ has a nontrivial bounded support.

Proof. If $\varrho \uparrow(0,+\infty)$ has a non-trivial bounded support, then clearly (2.2) holds with $p=1$ and

$$
\gamma=\operatorname{ess} \sup \varrho \uparrow(0, \infty)=\sup \{R ; R \text { in the support of } \varrho \uparrow(0, \infty)\}>0 .
$$

Conversely, assume first that $\varrho(0,+\infty)=0$. Then $\ln \int \exp (t x) \varrho(d x) \leqq 0$ for all $t \geqq 0$, and (2.2) cannot hold with $\gamma>0$.

Suppose now that the support of $\varrho \uparrow(0,+\infty)$ were not bounded, i.e. $\varrho[r, \infty)>0$ for all $r>0$. Then for all positive functions $f \in L^{1}(\varrho)$, we find that

$$
\int_{(-\infty, r)} f(x) \varrho_{t}(d x) \leqq \varrho([r, \infty))^{-1} \int_{(-\infty, r)} f(x) \exp (t(x-r)) \varrho(d x) \rightarrow 0
$$

with $t \rightarrow \infty$. In particular,

$$
\varrho_{t}[r, \infty)=1-\varrho_{t}(-\infty, r) \underset{t \rightarrow \infty}{\longrightarrow} 1 .
$$

Now by (2.6) and (2.7) for any $r>0,0<\varepsilon<1 / 2 r$,

$$
\begin{aligned}
\int x \varrho_{t}(d x) & =-\int_{(-\infty, 0)}|x| \varrho_{t}(d x)+\int_{[0, r)} x \varrho_{t}(d x)+\int_{[r, \infty)} x \varrho_{t}(d x) \\
& \geqq-\varepsilon+r(1-\varepsilon) \geqq r-1
\end{aligned}
$$

for sufficiently large $t$. Hence $\int x \varrho_{t}(d x) \rightarrow+\infty$ for $t \rightarrow \infty$, and (2.4) or equivalently (2.2) cannot hold with $0<\gamma<+\infty$ and $p=1$.

We shall now give another important class of measures $\varrho$ satisfying the condition (A), but where $p \neq 1$.

Proposition 2.2. Let

$$
P(x)=\sum_{k=1}^{q} b_{k} x^{k}
$$


with $b_{q}>0$ and $q$ an even integer, $\geqq 2$. Define

$$
\varrho(d x)=C^{-1} \exp (-P(x)) d x,
$$

where $C=\int \exp (-P(x)) d x<+\infty$. Then @ satisfies the condition $(\mathrm{A})$ with $p$ given $b y$

$$
\frac{1}{p}+\frac{1}{q}=1
$$

and

$$
\gamma=p^{-1}\left(q b_{q}\right)^{-(p-1)}>0
$$

Proof. By (2.4), we have to show that

$$
\lim _{t \rightarrow \infty} t^{-(p-1)} \int x \varrho_{t}(d x)=p \gamma=\left(q b_{q}\right)^{-(p-1)} .
$$

For $t \geqq 0$ and $x \in \mathbb{R}$, we define

$$
\tau=\left(t / q \cdot b_{q}\right)^{p-1}, \text { and } y=x-\tau,
$$

such that with $q-1=1 /(p-1)$, we have

$$
q b_{q} \tau^{q-1}=t
$$

We make the following substitutions

$$
\begin{aligned}
P(x)-t x & =b_{q}(x-\tau)^{q}-b_{q} \tau^{q}-b_{q} \sum_{i=2}^{q-1}\left(\begin{array}{l}
q \\
i
\end{array}\right)(-1)^{i} x^{i} \tau^{q-i}+\sum_{i=1}^{q-1} b_{i} x^{i} \\
& =b_{q} y^{q}-b_{q} \tau^{q}-b_{q} \sum_{i=2}^{q-1}\left(\begin{array}{l}
q \\
i
\end{array}\right)(-1)^{i}(y+\tau)^{i} \tau^{q-i}+\sum_{i=1}^{q-1} b_{i}(y+\tau)^{i} \\
& =P(y)+Q(\tau)-b_{q} \sum_{i=2}^{q-1} \sum_{l=0}^{i-1}\left(\begin{array}{l}
q \\
i
\end{array}\right)\left(\begin{array}{l}
i \\
l
\end{array}\right)(-1)^{i} y^{i-l} \tau^{q-i+l} \\
& +\sum_{i=2}^{q-1} \sum_{l=1}^{i-1} b_{i}\left(\begin{array}{l}
i \\
l
\end{array}\right) y^{i-l} \tau^{l}
\end{aligned}
$$

where we set

$$
Q(\tau)=-(q-1) b_{q} \tau^{q}+\sum_{l=1}^{q-1} b_{l} \tau^{l}
$$

using the fact that $1+\sum_{i=2}^{q-1}\left(\begin{array}{l}q \\ i\end{array}\right)(-1)^{i}=q-1$. In the third expression of $(2.15)$, the term with power $\tau^{q-1}$ has the coefficient $-b \sum_{q} \sum_{i=2}^{q-1}\left(\begin{array}{l}q \\ i\end{array}\right) i(-1)^{i} y \equiv 0$, while the fourth expression does not have terms of power $\tau^{q-1}$. The terms with the power $\tau^{q-2}$ in the two last expressions of (2.15) have together the coefficient

$$
-b_{q} \sum_{i=2}^{q-1}\left(\begin{array}{c}
q \\
i
\end{array}\right)\left(\begin{array}{c}
i \\
i-2
\end{array}\right)(-1)^{i} y^{2}+b_{q-1}(q-1) y=b_{q} q(q-1) y^{2} / 2+b_{q-1}(q-1) y \text {. (2. }
$$

Thus we can rewrite $(2.15)$ as

$$
P(x)-t x=Q(\tau)+\tau^{q-2}\left[b_{q} q(q-1) y^{2} / 2+b_{q-1}(q-1) y+R(y, \tau)\right],
$$


where $R$ has the form

$$
R(y, \tau)=\sum_{j=1}^{q-2} \sum_{l=1}^{q} a_{j l} y^{l} \tau^{-j},
$$

with suitable coefficients $\alpha_{j l}$. Set

$$
\tilde{C}=\int \exp \left\{-\tau^{q-2}\left[b_{q} q(q-1) y^{2} / 2+b_{q-1}(q-1) y+R(y, \tau)\right]\right\} d y .
$$

Then

$$
\begin{aligned}
t^{-(p-1)} \int x \varrho_{t}(d x) & =\left(q b_{q}\right)^{-(p-1)} \tau^{-1} \int(y+\tau) \exp \{\ldots\} d y / \tilde{C} \\
& =\left(q b_{q}\right)^{-(p-1)}\left[1+\tau^{-1} \int y \exp \{\ldots\} d y / \tilde{C}\right],
\end{aligned}
$$

where in $\{\ldots\}$ we have to repeat the argument of the exponential term in (2.20). Now when $t$ tends to infinity, then also $\tau \rightarrow \infty$, and the second term in the [ ]-bracket of (2.21) tends to zero. Hence

$$
\lim _{t \rightarrow \infty} t^{-(p-1)} \int x \varrho_{t}(d x)=\left(q b_{q}\right)^{-(p-1)}=p \gamma
$$

which is (2.12). The proposition is proven.

Remark. The algebraic transformations in the preceding proof are essentially involved in the proofs of Derrida's results [2]. Further on, however, we shall avoid these calculations and use instead the theory of large deviations and Laplace's method.

In our context, the interesting measures $\varrho$ are those which satisfy the condition (A) with $p>1$. By Proposition 2.1, they do not have a bounded support on $[0, \infty)$. The next theorem gives another characterisation for these measures.

Theorem 2.3. The probability measure $\varrho$ satisfies the condition (A) with $p>1$, if and only if

$$
b:=-\lim _{t \rightarrow \infty} t^{-p} \ln \varrho\left[t^{p-1}, \infty\right) \in(0, \infty) .
$$

If either of these conditions holds, then $\gamma$ from (2.2) and $b$ from (2.22) are related by the equation

$$
\gamma=p^{-1}(q b)^{-(p-1)}
$$

where $q$ is dual to $p$ in the sense of (2.10).

The somewhat lengthy proof of Theorem 2.3 is given in Sect. 12.

In Sect. 7, we shall use the following consequence of Theorem 2.3:

Corollary 2.4. Let $\varrho$ satisfy condition (A) with $p>1$ and let

$$
1<p_{1}<p<p_{2}<\infty \text {. }
$$

Let $q, q_{1}$, and $q_{2}$ denote the dual numbers of $p, p_{1}$, and $p_{2}$, respectively such that $1<q_{2}<q<q_{1}<\infty$. Then, for all $c_{1}, c_{2}>0$, we have

$$
\lim \sup _{x \rightarrow \infty}\left\{\ln \varrho[x, \infty)+c_{1} x^{q_{1}}\right\}=+\infty,
$$


and

$$
\lim _{x \rightarrow \infty}\left\{\ln \varrho[x, \infty)+c_{2} x^{q_{2}}\right\}=-\infty .
$$

Proof. Let $c_{1}, c_{2}>0$, and $0<\varepsilon<b$, where $b \in(0, \infty)$ is taken from (2.22). By (2.22), we find arbitrarily large $\bar{x}$ with

$$
\ln \varrho[\bar{x}, \infty) \geqq-(b+\varepsilon) \bar{x}^{q},
$$

and finally for all large $x$,

$$
\ln \varrho[x, \infty) \leqq-(b-\varepsilon) x^{q} .
$$

Hence, for those $\bar{x}$ and $x$, respectively, we have

$$
\ln \varrho[\bar{x}, \infty)+c_{1} \bar{x}^{q_{1}} \geqq c_{1} \bar{x}^{q_{1}}-(b+\varepsilon) \bar{x}^{q} \underset{x \rightarrow+\infty}{\longrightarrow}+\infty,
$$

and

$$
\ln \varrho[x, \infty)+c_{2} x^{q_{2}} \leqq-(b-\varepsilon) x^{q}+c_{2} x^{q_{2}} \underset{x \rightarrow \infty}{\longrightarrow}-\infty .
$$

This proves (2.25) and (2.26).

For the rest of the paper, we assume that the probability measure $\varrho$ satisfies condition (A).

\section{The Phase Transition of the Mean}

In this section we present the first result of this paper (Theorem 3.1): the asymptotic behaviour of the mean of $N^{-1} \ln Z_{N}, \lim N^{-1} E \ln Z_{N}$. Here $Z_{N}=Z_{N}(\beta, \varrho, \kappa)$ is taken from (1.2), where the $\zeta_{i}$ are independent random variables with distribution $\varrho$, and $\varrho$ satisfies the hypothesis (A) from Sect. 2. The proof of Theorem 3.1 is postponed to Sect. 8 .

We start with the following definitions:

$$
\beta_{C}(\varrho, z)=\left\{\begin{array}{lll}
+\infty & \text { for } & p=1 \\
((q-1) z / \gamma)^{1 / p} & \text { for } & p>1
\end{array}\right.
$$

where $p$ and $\gamma$ are given by (2.2) and $q$ is dual to $p$ in the sense of (2.10).

$$
\psi(\beta, \varrho, z)= \begin{cases}0 & \text { for } 0<\beta \leqq \beta_{C}(\varrho, z), \\ \gamma\left[p \beta_{C}^{p-1}(\varrho, z) \beta-\beta^{p}\right]-z & \text { for } \quad \beta_{C}(\varrho, z)<\beta \text { and } p>1 .\end{cases}
$$

We have $\psi(\beta, \varrho, z) \leqq 0$ with equality exactly for $0<\beta \leqq \beta_{C}(\varrho, z)$.

It is easy to check that for $p>1, \psi$ is - as a function of $\beta$-differentiable at $\beta_{C}=\beta_{C}(\varrho, z)$ with

$$
\psi\left(\beta_{C}, \varrho, z\right)=0,
$$

and

$$
\lim _{\beta \rightarrow \beta_{C}}\left(\frac{d}{d \beta} \psi\right)(\beta)=0
$$




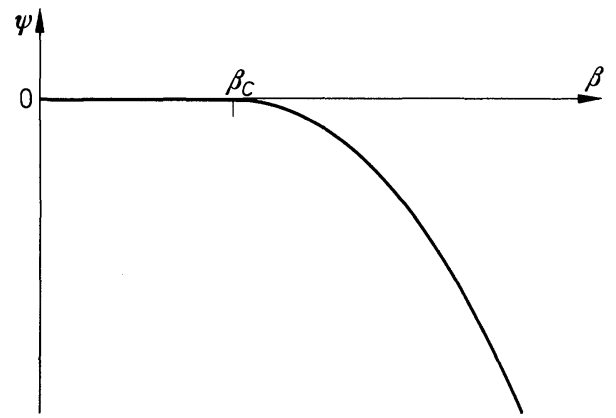

Fig. 1. The function $\beta \mapsto \psi(\beta, \varrho, z)$ for $p>1$

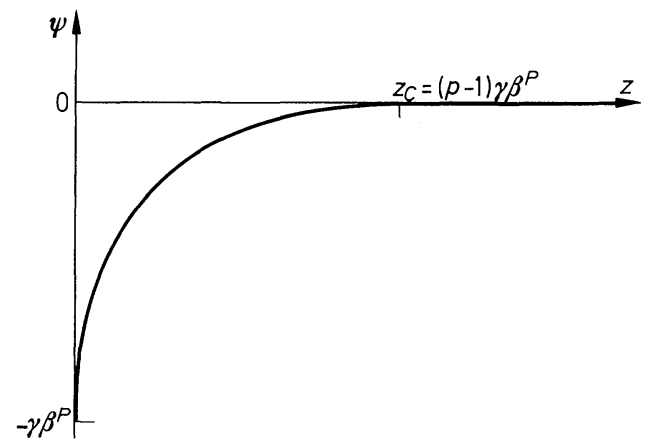

Fig. 2. The function $z \mapsto \psi(\beta, \varrho, z)$ for $p>1, \beta>\beta_{C}$

while

$$
\lim _{\beta \backslash \beta_{C}}\left(\frac{d^{2}}{d \beta^{2}} \psi\right)(\beta)=-\gamma p(p-1) \beta_{C}^{p-2}<0
$$

In the standard Gaussian case, $\beta_{C}$ and the function $\psi$ are given by the righthand side of (1.6). The generalization of (1.6) is now:

Theorem 3.1. If $\varrho$ satisfies hypothesis (A), then

$$
\lim _{N \rightarrow \infty} N^{-1} E \ln Z_{N}(\beta, \varrho, \kappa)=\psi(\beta, \varrho, \ln \kappa) .
$$

Remark. In the Curie-Weiss model without external field, the specific energy is a function which is continuous, but in general not continuously differentiable at the critical Curie temperature. This is called a second-order phase transition.

In comparison with this, the result of Theorem 3.1 may be called a phase transition of third order, since the mean energy $\psi$ is once, but not twice, continuously differentiable at the critical temperature. (The definition of "first-" and "second-order" phase transition, given by Dyson [3, p. 8], corresponds in our terminology to "first-" and "second- or higher-order" phase transition.) 


\section{Hypergeometric Bound from below and a Hypergeometric Entropy Function}

In the preceding section, we showed that the expectation of the normalized partition function $N^{-1} \ln Z_{N}(\beta, \varrho, \kappa)$ converges to $\psi(\beta, \varrho, \ln \kappa)$. But in fact, we even have convergence in probability of the random variable $N^{-1} \ln Z_{N}(\beta, \varrho, \kappa)$ to the non-random function $\psi=\psi(\beta, \varrho, \ln \kappa)$. To show this, we shall first prove that

$$
\text { " } N^{-1} \ln Z_{N} \text { is hypergeometrically bounded from below by } \psi \text {." }
$$

To explain this phrase, we first remind the reader of the notion of geometric onesided boundedness :

Definition 4.1. (i) A sequence of random variables $Y_{N}$ with normalizing constants $a_{N}, a_{N} \rightarrow \infty$, is geometrically bounded from below (respectively, above) by $\alpha$, if for all $\varepsilon>0$ there exists a $\delta>0$ such that

$$
\begin{aligned}
& \underset{N}{\limsup a_{N}{ }^{-1}} \ln \operatorname{Prob}\left\{Y_{N} / a_{N} \leqq \alpha-\varepsilon\right\} \leqq-\delta \\
&\left(\text { respectively, } \limsup _{N} a_{N}^{-1} \ln \operatorname{Prob}\left\{Y_{N} / a_{N} \geqq \alpha+\varepsilon\right\} \leqq-\delta\right) .
\end{aligned}
$$

(ii) The sequence $Y_{N} / a_{N}$ converge geometrically to $\alpha$, if $\left(Y_{N}, a_{N}\right)$ is geometrically bounded by $\alpha$ simultaneously from below and from above. (See also [4, Definition II, 2.1].)

In part (i) of the definition, Prob \{\} denotes the probability of the event written in the brackets. Informally, (4.1) means that

$$
0 \leqq \operatorname{Prob}\left\{Y_{N} / a_{N} \leqq \alpha-\varepsilon\right\} \leqq \exp \left(-a_{N} \delta\right)
$$

for all large $N$. In many cases, geometric bounds are obtained from the entropy function to the sequence $\left(Y_{N}, a_{N}\right)$.

For a subset $A \subseteq \mathbb{R}$, define

$$
\hat{i}_{1}(A)=-\lim \sup a_{N}^{-1} \ln \operatorname{Prob}\left\{Y_{N} / a_{N} \in A\right\} \geqq 0,
$$

and then for $x \in \mathbb{R}$

$$
\hat{i}_{1}(x)=\sup i_{1}(A),
$$

where the supremum is taken over all intervals $A \subseteq \mathbb{R}$, containing $x$ in their interior. Finally, let

$$
i_{1}(x)=\operatorname{clc}\left(\hat{i}_{1}\right)(x),
$$

where $\operatorname{clc}\left(\hat{i}_{1}\right)$ denotes the largest closed (=lower semicontinuous) convex function majorized by $\hat{i}$. Then $i_{1}$ is called the limiting entropy function of $\left(Y_{n}, a_{n}\right)$. One can show that $i_{1}$ is the Legendre transform of the limiting $\Phi$-function $\varphi_{1}$ of $\left(Y_{n}, a_{n}\right)$

$$
\varphi_{1}(u)=\lim _{N} a^{-N} \ln E \exp \left(t Y_{N}\right)
$$

The Legendre transformation $\varphi_{1}^{*}$ of $\varphi_{1}$ is given by

$$
\varphi_{1}^{*}(x)=\sup _{u}\left\{x u-\varphi_{1}(u)\right\} \text {. }
$$


We then have

$$
\varphi_{1}=\left(\hat{i}_{1}\right)^{*}, \text { and } i_{1}=\varphi_{1}^{*}=\hat{i}_{1}^{* *} .
$$

We tacitly assume here that $\varphi_{1}$ exists and is finite at least on an open, non-empty interval. Then $\alpha$ is a geometric lower bound for $\left(Y_{N}, a_{N}\right)$ in the sense of (4.1) if and only if for all $\varepsilon>0$

$$
\inf _{x \leqq \alpha-\varepsilon} i_{1}(x)=\delta>0 .
$$

Similarly for a geometric upper bound.

In analogy to Definition 4.1, we introduce the notion of a hypergeometric bound.

Definition 4.2. The random variables $Y_{N}$ with normalization $a_{N}, a_{N} \rightarrow \infty$, are hypergeometrically bounded from below (respectively, above) by $\alpha$, if for all $\varepsilon>0$ there exists $\delta>0$ with

$$
\begin{gathered}
\liminf a_{N}^{-1} \ln \left(-\ln \operatorname{Prob}\left\{Y_{N} / a_{N} \leqq \alpha-\varepsilon\right\}\right) \geqq \delta \\
\left(\text { respectively, } \liminf a_{N}^{-1} \ln \left(-\ln \operatorname{Prob}\left\{Y_{N} / a_{N} \geqq \alpha+\varepsilon\right\}\right) \geqq \delta\right) .
\end{gathered}
$$

Inequality (4.10) means informally

$$
0 \leqq \operatorname{Prob}\left\{Y_{N} / a_{N} \leqq \alpha-\varepsilon\right\} \leqq \exp \left(-\exp \left(a_{N} \delta\right)\right)
$$

Of course, (4.10) is much stronger than (4.1).

In analogy to part (ii) of Definition 4.1, one could also introduce the definition of hypergeometric convergence. But since we do not know natural examples of hypergeometric convergence at the moment, this would be a purely abstract definition. We shall however introduce the definition of the hypergeometric entropy function to $\left(Y_{N}, a_{N}\right)$ :

$$
\hat{i}_{2}(A)=\liminf a_{N}^{-1} \ln \left(-\ln \operatorname{Prob}\left\{Y_{N} / a_{N} \in A\right\}\right),
$$

and

$$
\hat{i}_{2}(x)=\sup \hat{i}_{2}(A),
$$

where again the supremum is taken over all intervals $A$ containing $x$ in their interior. The interesting region is of course $\left\{x, \hat{i}_{2}(x)>0\right\}$. For our problem $\left(\ln Z_{N}, N\right)$, Theorem 4.3 below will give a positive lower bound for $\hat{i}_{2}(x)$ in the region $(-\infty, \psi(\beta, \varrho, \ln \kappa))$. At the moment, we do not know a natural definition of a hypergeometric $\Phi$-function $\varphi_{2}$, nor do we know what kind of relation should hold between $\varphi_{2}$ and $i_{2}$ in analogy to (4.6)-(4.8).

Let us define for $q \in(-\infty, 0)$

$$
j(q)=j(q, \beta, \varrho)= \begin{cases}\ln \kappa & \text { if } p=1, q \leqq 0 \\ \ln \kappa & \text { if } p>1 \text { and } q<-\gamma \beta^{p} \\ \ln \kappa-\psi^{-1}(\beta, \varrho, q) & \text { if } p>1 \text { and }-\gamma \beta^{p} \leqq q \leqq 0\end{cases}
$$

where $\psi^{-1}(\beta, \varrho, \cdot)$ is the inverse function of $z \mapsto \psi(\beta, \varrho, z)$ on $\left[-\gamma \beta^{p}, 0\right)$ (see Fig. 3).

The following theorem states that $j$ is a lower bound for the hypergoemetric entropy function $\hat{i}_{2}$ of $\left(\ln Z_{N}, N\right)$. Its proof is given in Sect. 9. 


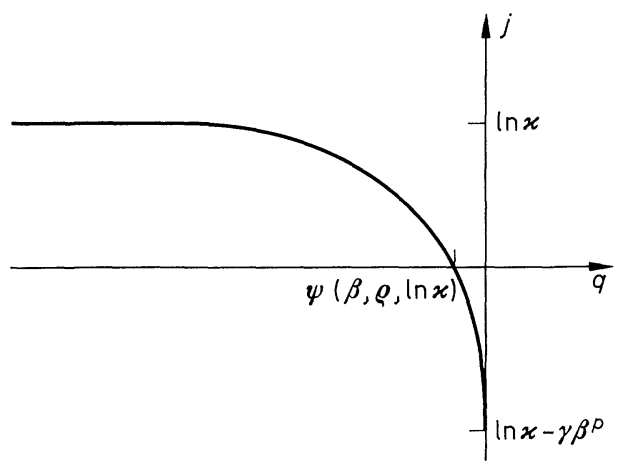

Fig. 3. The function $q \mapsto j(\beta, \varrho, q)$

Theorem 4.3. For all $q<0$ we have

$$
\liminf N^{-1} \ln \left(-\ln \operatorname{Prob}\left\{N^{-1} \ln Z_{N} \leqq q\right\}\right) \geqq j(\beta, \varrho, q) .
$$

Remarks. 1. Of course, (4.15) is only of interest in the region $q \in(-\infty, \psi(\beta, \varrho, \ln \kappa))$, where $j$ is positive.

2. There are some hints which indicate that the estimate in (4.15) is sharp, and that we can even replace lim inf by $\lim$. If this were true, we could say that $j$ is the hypergeometric entropy function for $\ln Z_{N}$ in the region $(-\infty, \psi(\beta, \varrho, \ln \kappa))$. However, we do not yet know how to prove the inverse inequality of (4.15).

3. The fact that the entropy function is of hypergeometric order in the region $(-\infty, \psi(\beta, \varrho, \ln \kappa))$, is obviously related to the iterated large deviation problem as described in the introduction [see (1.7)]. However, there does not seem to exist a simple cookbook recipe for boiling down an iterated large deviation problem to a hypergeometric entropy.

With Definition 4.2, we get as an immediate consequence of Theorem 4.3:

Corollary 4.4. The limit $\lim N^{-1} E \ln Z_{N}=\psi(\beta, \varrho, \ln \kappa)$ is a hypergeometric lower bound for the random variables $\ln Z_{N}$ with normalization $N$.

Since now the probabilities $\operatorname{Prob}\left\{N^{-1} \ln Z_{N} \leqq \psi(\beta, \varrho, \ln \kappa)-\varepsilon\right\}$ with $\varepsilon>0$ are summable, we have by the Borel-Cantelli lemma:

\section{Corollary 4.5.}

$$
\operatorname{Prob}\left\{\liminf N^{-1} \ln Z_{N} \geqq \psi(\beta, \varrho, \ln \kappa)\right\}=1
$$

\section{Convergence of $N^{-1} \ln Z_{N}$}

In the last section we have shown that $\psi(\beta, \varrho, \ln \kappa)$ is a hypergoemetric lower bound for $N^{-1} \ln Z_{N}$. We want to show that indeed $N^{-1} \ln Z_{N}$ converges at least stochastically to $\psi(\beta, \varrho, \ln \kappa)$. For this purpose, we need the following result:

\section{Lemma 5.1.}

$$
\lim _{N \rightarrow \infty} N^{-1} \ln \operatorname{Prob}\left\{N^{-1} \ln Z_{N} \geqq q\right\} \leqq-q
$$

for all q. 
Proof. We apply the inequality

$$
1_{[\alpha,+\infty)}(x) \leqq x / \alpha \quad \text { for } \quad x \geqq 0, \quad \alpha \geqq 0
$$

to $x=Z_{N}$ and $\alpha=\exp (N q)$. Thus

$$
1_{[q, \infty)}\left(N^{-1} \ln Z_{N}\right)=1_{[\exp (N q), \infty)}\left(Z_{N}\right) \leqq Z_{N} \cdot \exp (-N q),
$$

or

$$
\lim _{N \rightarrow \infty} N^{-1} \ln \operatorname{Prob}\left\{N^{-1} \ln Z_{N} \geqq q\right\} \leqq \lim _{N} N^{-1} \ln E\left(Z_{N}\right)-q=-q,
$$

since by (2.2)

$$
\lim _{N} N^{-1} \ln E\left(Z_{N}\right)=\beta^{p}\left[\lim _{N}\left(N^{1 / p} \beta\right)^{-p} \ln \int \exp \left(N^{1 / p} \beta x\right) \varrho(d x)-\gamma\right]=0 .
$$

The Borel-Cantelli lemma yields the simple consequence of Lemma 5.1.

\section{Corollary 5.2.}

$$
\operatorname{Prob}\left\{\lim \sup N^{-1} \ln Z_{N} \geqq 0\right\}=0 .
$$

Combining Corollary 4.4 and Lemma 5.1, we get almost sure convergence in the case $\beta \leqq \beta_{C}$.

Theorem 5.3. If $\beta \leqq \beta_{C}$, and in particular if $p=1$, then $N^{-1} \ln Z_{N}$ converges geometrically, and a fortiori, almost surely to $\psi(\beta, \varrho, \ln \kappa)=0$.

In the case of a phase transition, i.e. $\beta>\beta_{C}$ and $p>1$, we have a weaker form of convergence:

Theorem 5.4. If $\beta>\beta_{C}$, then $N^{-1} \ln Z_{N}$ converges stochastically to $\psi=\psi(\beta, \varrho, \ln \kappa)<0$.

Proof. The assertion of the theorem is known to be equivalent to the statement that the distributions of $N^{-1} \ln Z_{N}$ converge weakly to $\delta_{\psi}$. By Corollary 4.4 and Lemma 5.1, the distributions of $N^{-1} \ln Z_{N}$ are tight, and any limit probability measure $\mu$ has its support on $[\psi, 0]$. By Theorem 3.1, we have

$$
\int x \mu(d x)=\lim N^{-1} E \ln Z_{N}=\psi .
$$

Hence $\mu=\delta_{\psi}$, since $\delta_{\psi}$ is the only probability measure which has support on $[\psi, 0]$ and satisfies (5.7).

Remark. We believe that even in the case $\beta>\beta_{C}$ we have almost sure convergence of $N^{-1} \ln Z_{N}$ to $\psi(\beta, \varrho, \ln \kappa)$. In fact, it is known that by Theorem 5.4, we can find a subsequence $\left(N_{i}\right)$ of $(N)$ such that $\left(N_{i}^{-1} \ln Z_{N_{i}}\right)_{i}$ converges almost surely to $\psi$. By the special form of $Z_{N}$ as a sum of independent random variables depending on $N$, it is most plausible that not only the subsequence, but also the whole sequence $N^{-1} \ln Z_{N}$ converges almost surely. At the moment, however, we do not have a rigorous proof for the almost sure convergence. On the other hand, we believe that for $\beta>\beta_{C}$

$$
N^{-1} \ln Z_{N} \text { does not converge geometrically to } \psi,
$$

because we have a phase transition for $\beta>\beta_{C}$, and the entropy function vanishes in the interval $[\psi, 0]$. (See also Remark 1 at the end of Sect. 6.) 


\section{Discussion of the $\Phi$-Function and its Entropy}

Still, our main purpose is to compute the limit function $\Phi$

$$
\Phi(t)=\Phi(\beta, \varrho, \kappa)(t)=\lim \Phi_{N}(t)=\lim _{N} N^{-1} \ln E \exp \left\{t \ln Z_{N}(\beta, \varrho, \kappa)\right\} .
$$

All $\Phi_{N}$, and hence also $\Phi$, are convex functions. Once we know $\Phi$, we get the entropy function of $\left(\ln Z_{N}(\beta, \varrho, \kappa) ; N\right)$ as the Legendre transform of $\Phi$

$$
I(\beta, \varrho, \kappa)(q)=\sup _{t}(q \cdot t-\Phi(\beta, \varrho, \kappa)(t))=\Phi^{*}(\beta, \varrho, \kappa)(q) .
$$

For the case $p=1$, a complete calculation of $\Phi$ is given by the following theorem, the proof of which is postponed until Sect. 10.

Theorem 6.1. If $p=1$, then

$$
\Phi(\beta, \varrho, \kappa) \equiv 0,
$$

and

$$
I(\beta, \varrho, \kappa)(q)=\left\{\begin{array}{lll}
0 & \text { if } & q=0 \\
+\infty & \text { if } & q \neq 0 .
\end{array}\right.
$$

Remark. For $q<0$, the result (6.4) is a trivial consequence of Theorem 4.1, where we have even shown that the hypergeometric entropy function $J$ is greater than or equal to $\ln \kappa$. Informally, the relation between the entropy function $I$ and the hypergeometric entropy function $J$ is given by

$$
\text { “I } \approx \lim N^{-1} \exp (N J) " .
$$

If now $J \geqq \ln \kappa>0$ in the region $q<0$, then $I$ must be $+\infty$. At the moment, we do not know if $\ln Z_{N}$ also has a non-zero hypergoemetric entropy function in the region $q>0$, as it might be suggested by the theorem.

Now we turn to the more difficult problem, the computation of $\Phi$ for $p>1$. First, we give a description of what we believe that the function $\Phi$ looks like. Later we shall compute some of the values of $\Phi$. There are however some questions which remain unsolved.

Define for $t \geqq 1, p>1$, and $q$ dual to $p$ [see (2.10)]

$$
\chi(t)= \begin{cases}q-1 & \text { for } t=1 \\ (t-1) /\left(t^{p}-t\right) & \text { for } t>1\end{cases}
$$

Here $\chi$ is continuous and strictly decreasing with values in $(0, q-1]$.

From (3.1), we recall

$$
\beta_{C}=\beta_{C}(\varrho, \ln \kappa)=((q-1) \ln \kappa / \gamma)^{1 / p} .
$$

Put

$$
t(\beta)= \begin{cases}\chi^{-1}\left((q-1)\left(\beta / \beta_{C}\right)^{p}\right) & \text { for } \beta \leqq \beta_{C} \\ 1 & \text { for } \beta>\beta_{C} .\end{cases}
$$


Finally, we define

$$
\hat{\Phi}(t)=\hat{\Phi}(\beta, \varrho, \kappa)(t)= \begin{cases}t \cdot \psi(\beta, \varrho, \ln \kappa) & \text { for } t \leqq 0, \\ 0 & \text { for } 0 \leqq t \leqq t(\beta), \\ \ln \kappa\left(t^{p}-t\right)\left[(q-1)\left(\beta / \beta_{C}\right)^{p}-\chi(t)\right] & \text { for } t \geqq t(\beta) .\end{cases}
$$

In Fig. 5a and b, the dotted curves are the analytic extension of $\hat{\Phi} \uparrow(t(\beta),+\infty)$. For $\beta>\beta_{C}$, the function $t \mapsto t \cdot \psi(\beta, \varrho, \ln \kappa)$ is just the tangent at $t=\beta_{C} / \beta$ of the analytic extension of $\hat{\Phi} \uparrow(1, \infty)$ which passes through zero.

We remark that for all $\beta$

$$
\hat{\Phi}^{\prime}(t(\beta)+)=\lim _{t \downarrow t(\beta)} \hat{\Phi}^{\prime}(t)=\ln \kappa\left[\left(\frac{p t(\beta)^{p-1}-1}{p-1}\right)\left(\beta / \beta_{C}\right)^{p}-1\right],
$$

which is equal to zero exactly for $\beta=\beta_{C}(\varrho, \ln \kappa)$.

As the notation already indicates, we have the following

Conjecture. For $p>1$,

$$
\Phi(\beta, \varrho, \kappa)(t):=\lim _{N} \Phi_{N}(\beta, \varrho, \kappa)(t)=\hat{\Phi}(\beta, \varrho, \kappa)(t) .
$$

Unfortunately, we do not know how to prove (6.11) for all $t$. All that we can do is the following partial result, which is proved in Sect. 11.

Theorem 6.2. Let $p>1$. Then $\Phi(\beta, \varrho, \kappa)(t)=\hat{\Phi}(\beta, \varrho, \kappa)(t)$ for $t \in(-\infty, 0] \cup\{1,2,3, \ldots\}$.

Remarks. 1. Evidently Theorem 6.2 makes the conjecture $\Phi=\hat{\Phi}$ for all $t$ rather plausible. However, there remain some doubts, in particular in the case $\beta>\beta_{C}$ and $t \in(0,1)$, where $\Phi$ could also be negative. We do not know how to settle this question.

2. Let $\hat{I}=\hat{I}(\beta, \varrho, \kappa)$ be the Legendre transform of $\hat{\Phi}$, i.e. by $(6.2)$

$$
\hat{I}(\beta, \varrho, \kappa)=(\hat{\Phi}(\beta, \varrho, \kappa))^{*} .
$$

$\hat{I}$ has roughly the following shape:

$$
\hat{I}(q)=\left\{\begin{array}{lll}
+\infty & \text { for } & q<\psi(\beta, \varrho, \ln \kappa), \\
0 & \text { for } & \psi(\beta, \varrho, \kappa) \leqq q \leqq 0 \\
\text { in }(0, \infty) & \text { for } & 0<q
\end{array}\right.
$$

Under the conjecture $\Phi=\hat{\Phi}$, the function $\hat{I}$ equals the entropy function of $N^{-1} \ln Z_{N}$, i.e. informally

$$
\operatorname{Prob}\left\{N^{-1} \ln Z_{N} \in d q\right\} \approx \exp (-N \hat{I}(q))
$$

[see also (4.3)-(4.5)]. The fact that $\hat{I}(q)=+\infty$ for $q<\psi(\beta, \varrho, \ln \kappa)$, is consistent with Theorem 4.3, where we have shown that there exists even an hypergeometric entropy function. Similarly, Lemma 5.1 gives a lower bound for $\hat{I}$ in the region $q>0$ :

$$
\hat{I}(q) \geqq q \quad \text { for } \quad q>0 .
$$

Finally, let $\beta>\beta_{C}$. The existence of the non-empty interval $[\psi(\beta, \varrho, \ln \kappa), 0]$ on which $\hat{I}$ vanishes, is the usual mathematical phenomenon which occurs in the case of a phase transition. 


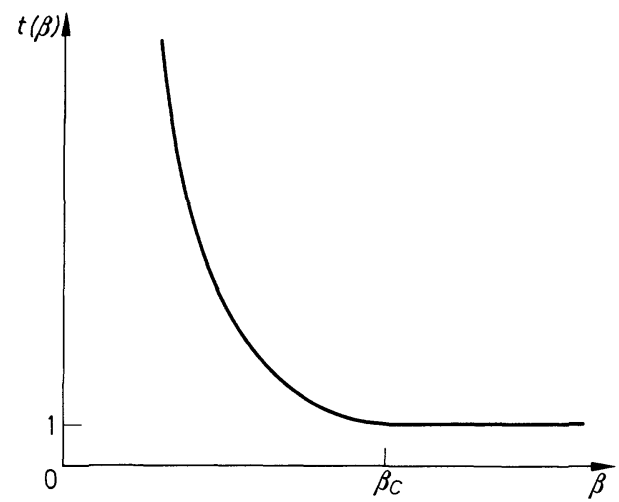

Fig. 4. The function $t=t(\beta)$
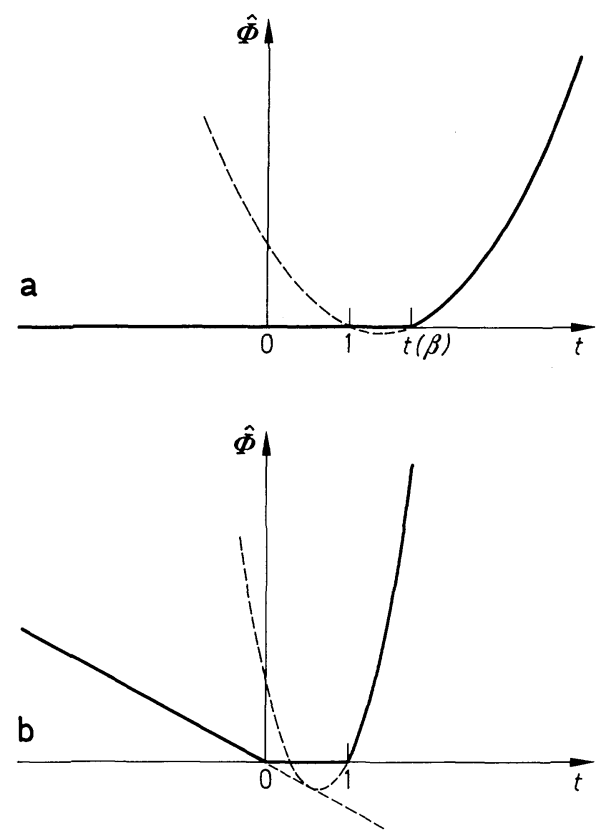

Fig. 5a and b. The function $\hat{\Phi}=\hat{\Phi}(t)$, for $\beta<\beta_{C}$ (a), and for $\beta>\beta_{C}$ (b)

3. The random variable $\ln Z_{N}$ has a hypergeometric entropy function for $q>\psi(\beta, \varrho, \ln \kappa)$, while for $q>0$ the usual entropy function is positive and finite. This indicates that the central limit theorem cannot hold for $\ln Z_{N}$ with any suitable normalization.

4. As in Theorem 6.2, also in the Sherrington-Kirkpatrick model, one is only able to calculate the "replicas" $\Phi(n)$ for $n=0,1,2, \ldots$ If now the points $(0, \Phi(0))$, $(1, \Phi(1))$, and $(2, \Phi(2))$ are collinear in $\mathbb{R}^{2}$, then $\Phi(q)=q \cdot \Phi(1)+\Phi(0)$ for $q \in[0,2]$ and $\Phi^{\prime}(0+)=\Phi(1)$. This is essentially the replica method. However, this does not give 
any information at all about $\Phi^{\prime}(0-)$, which in our model turns out to be the important value, since the random variables $N^{-1} \ln Z_{N}$ converge to it. But the remark after (6.9) gives another method for computing $\Phi^{\prime}(0-)$ from the replica values $\Phi(n), n=1,2, \ldots$. For $\beta \geqq \beta_{C}, \Phi^{\prime}(0-)$ is exactly the slope of the tangent to the analytical extension of $(\Phi(n))_{n}$, which passes through zero. It may be that a similar procedure also works in the SK model. In fact, the above result confirms and substantiates an older idea of van Hemmen, Palmer, and Sommers, who proposed to take the convex hull of the "analytic" continuation of $\Phi(n)$, including $\Phi(0)$. However, numerical data (Palmer, unpublished) were not in favour of this proposal (private communication by J. L. van Hemmen).

\section{The Random Variable $\exp \left(N^{1 / p} \beta \zeta-N \gamma \beta^{p}\right)$}

Before we give a proof of Theorem 3.1, we shall first investigate the random variable

$$
\eta=\eta(N, \beta, \varrho)=\exp \left(N^{1 / p} \beta \zeta-N \gamma \beta^{p}\right)
$$

its $\Phi$-function and its entropy function. Here $\zeta$ is a random variable with distribution $\varrho, p \in[1, \infty)$, and $\gamma \in(0, \infty)$ stem from the condition (A) of Sect. 2.

In particular, we are interested in the asymptotic behaviour of $\eta$ for large $N$. First, let us define the $\Phi$-function of $\eta$ :

$$
\varphi_{N}(u)=\varphi_{N}(\beta, \varrho, u)=\ln \int \exp \left(u \cdot \exp \left(N^{1 / p} \beta x-N \gamma \beta^{p}\right)\right) \varrho(d x) .
$$

The $\Phi$-function is always convex (cf. [4]). Unfortunately, in our case the functions $\varphi_{N}$ are degenerate for $p>1$. More precisely, we have the following

Theorem 7.1. Assume that $p>1$. Then

$$
\varphi_{N}(u)=\left\{\begin{array}{lll}
+\infty & \text { for } & u>0 \\
0 & \text { for } & u=0 \\
\text { in }(-\infty, 0) & \text { for } & u<0
\end{array}\right.
$$

On $(-\infty, 0)$, the function $u \mapsto \varphi_{N}(u)$ is strictly increasing, convex and real analytic. Moreover,

$$
\lim _{u \uparrow 0}\left(\frac{d}{d u} \varphi_{N}\right)(u)=\int \varrho(d x) \exp \left(N^{1 / p} \beta x-N \gamma \beta^{p}\right)<+\infty
$$

Proof. We shall first prove $\varphi_{N}(u)=+\infty$ for $u>0$. Let again $q$ denote the dual of $p$ and $q_{1}>q$. For any $R>0$, we find by (2.28) arbitrarily large $\bar{x}$ with

$$
\ln \varrho[\bar{x}, \infty)+u \bar{x}^{q_{1}} \geqq R,
$$

whence for all $x \leqq \bar{x}$

$$
u \exp \left(N^{1 / p} \beta x\right)+\ln \varrho[x, \infty) \geqq R+u\left(\exp \left(N^{1 / p} \beta x\right)-\bar{x}^{q_{1}}\right)
$$


By partial integration, we get

$$
\begin{aligned}
& \int \exp \left(u \exp \left(N^{1 / p} \beta x-N \gamma \beta^{p}\right)\right)(d x) \\
& \quad=1+u N^{1 / p} \beta \int \exp \left\{N^{1 / p} \beta x-N \gamma \beta^{p}+u \exp \left(N^{1 / p} \beta x-N \gamma \beta^{p}\right)+\ln \varrho[x, \infty)\right\} d x \\
& \quad \geqq 1+u N^{1 / p} \beta \int_{-\infty}^{\bar{x}} \exp \left\{N^{1 / p} \beta x-N \gamma \beta^{p}+R+u\left(\exp \left(N^{1 / p} \beta x-N \gamma \beta^{p}\right)-\bar{x}^{q_{1}}\right\} d x\right. \\
& \quad \rightarrow+\infty \text { for } \bar{x} \rightarrow \infty .
\end{aligned}
$$

This shows $\varphi_{N}(u)=+\infty$ for all $u>0$. That $\varphi_{N}(0)=0$ and $\varphi_{N}(u)<0$ for $u<0$ are evident. So is the fact that $\varphi_{N}$ is strictly increasing and real analytic on $(-\infty, 0)$. Finally, under the condition (2.1), Eq. (7.4) is an immediate consequence of Lebesgue's theorem.

Digression. Many theorems in the theory of large deviation are stated under the general hypothesis that the (limit) $\Phi$-function $\varphi(t)$ is a finite, convex function in a neighborhood of 0 (see [4, Hypothesis II.1.1]). Theorem 7.1 now yields natural examples of free energy functions which do not satisfy this hypothesis. We shall briefly point out some of the consequences. For the moment, we keep all the parameters $N, \beta, \varrho$ fixed and drop the corresponding subscripts. We only assume the conditions of Theorem 7.1 to hold. Let $\eta_{1}, \eta_{2}, \ldots$ be independent copies of the random variable $\eta(6.1)$. Set

$$
Y_{n}=\sum_{i=1}^{n} \eta_{i}
$$

Of course, the $\Phi$-function of $Y_{n}$,

$$
\varphi(u)=n^{-1} \ln E \exp \left(u Y_{n}\right)
$$

is independent of $n$ and equal to $\varphi_{N}(u)$ from (7.2). It does not satisfy the above hypothesis. Moreover, $\varphi$ is not even maximal (cf. [4, p. 13]), since

$$
\tilde{\varphi}(u)=\left\{\begin{array}{lll}
\varphi(u) & \text { for } & u \leqq 0 \\
u \cdot \varphi^{\prime}(0-) & \text { for } & u>0
\end{array}\right.
$$

is a convex extension of $\varphi \uparrow\{u, \varphi(u)<+\infty\}$. Here $\varphi^{\prime}(0-)$ means the left-hand limit of $\varphi^{\prime}(u)$ at zero. By the law of large numbers, we know that

$$
n^{-1} Y_{n} \underset{\text { a.s. }}{\Rightarrow} E(\eta)=\int \varrho(d x) \exp \left(N^{1 / p} \beta x-N \gamma \beta^{p}\right)=\varphi^{\prime}(0-),
$$

where $\underset{\text { a.s. }}{\Rightarrow}$ denotes almost sure convergence.

However, the entropy function $i$ of $\eta$

$$
i(z)=\sup _{u}\{u z-\varphi(u)\},
$$

which is given as the Legendre transform of $\varphi$, vanishes for $z \geqq E(\eta)=\varphi^{\prime}(0-)$ and is positive otherwise:

$$
i(z)\left\{\begin{array}{lll}
>0 & \text { for } \quad z<\varphi^{\prime}(0-), \\
=0 & \text { for } \quad z \geqq \varphi^{\prime}(0-) .
\end{array}\right.
$$


Since for nice sets $A$

$$
\lim n^{-1} \ln \operatorname{Prob}\left\{n^{-1} Y_{n} \in A\right\}=-\inf _{z \in A} i(z),
$$

we find that $n^{-1} Y_{n}$ converges even geometrically from below to $\varphi^{\prime}(0-)$, i.e. $\left(Y_{n}, n\right)$ is geometrically bounded from below by $\varphi^{\prime}(0-)$. But $n^{-1} Y_{n}$ does not converge geometrically from above to $\varphi^{\prime}(0-)$. Thus, Theorem 7.1 gives a natural example where we have almost sure convergence, but not the stronger result of geometric convergence. A similar discussion has been given in the fundamental paper of Lanford (see [7, pp. 47-49]).

The singularities of the functions $\varphi_{N}(u)$, as stated in Theorem 7.1, force us to look for more suitable functions which incorporate the relevant features of the random variable $\eta$. We make the following proposal:

Definition 7.2. For $t \in \mathbb{R}$, let

$$
g_{N}(t)=g_{N}(t, \beta, \varrho)=\int_{0}^{\infty} d u e^{-u} \varrho\left[\left(\ln u+N\left(t+\gamma \beta^{p}\right)\right) / \beta N^{1 / p}, \infty\right),
$$

where again $\gamma \in(0,+\infty)$ and $p \geqq 1$ are given by (2.2).

The following equality shows the connection between $g_{N}$ and the $\Phi$-function $\varphi_{N}:$

Lemma 7.3.

$$
\ln \left(1-g_{N}(t)\right)=\varphi_{N}(-\exp (-N t))
$$

Proof. By partial integration and substitution $u=\exp \left(N^{1 / p} \beta x-N\left(t+\gamma \beta^{p}\right)\right)$, we get

$$
\begin{aligned}
1-g_{N}(t) & =\int_{0}^{\infty} d u e^{-u} \varrho\left(-\infty,\left(\ln u+N\left(t+\gamma \beta^{p}\right)\right) / \beta N^{1 / p}\right) \\
& =\int \varrho\left(d\left(\left(\ln u+N\left(t+\gamma \beta^{p}\right)\right) / \beta N^{1 / p}\right) e^{-u}\right. \\
& =\int \varrho(d x) \exp \left(-\exp \left(N^{1 / p} \beta x-N\left(t+\gamma \beta^{p}\right)\right)\right) \\
& =\exp \left(\varphi_{N}(-\exp (-N t))\right),
\end{aligned}
$$

which is just (7.16).

Let us rewrite (7.17) as

$$
g_{N}(t)=\int \varrho(d x)\left[1-\exp \left(-\exp \left(N^{1 / p} \beta x-N\left(t+\gamma \beta^{p}\right)\right)\right)\right] .
$$

We collect some simple properties of $g_{N}(t)$, which follow directly from the definition or (7.18).

Lemma 7.4. (i) $g_{N}$ is real analytic and strictly decreasing. Moreover

for all $t \in \mathbb{R}$.

$$
1=\lim _{t^{\prime} \rightarrow-\infty} g_{N}\left(t^{\prime}\right)>g_{N}(t)>\lim _{t^{\prime} \rightarrow+\infty} g_{N}\left(t^{\prime}\right)=0
$$

(ii) For $p=1$, we have

$$
\begin{array}{r}
\lim _{N \rightarrow \infty} g_{N}(t)=\varrho(t / \beta+\gamma, \infty)+\varrho\{t / \beta+\gamma\} \cdot\left(1-e^{-1}\right), \\
\lim _{N \rightarrow \infty} g_{N}(t)=\left\{\begin{array}{lll}
1 & \text { if } & t<-\gamma \beta^{p}, \\
\varrho(0, \infty)+\varrho\{0\}\left(1-e^{-1}\right) & \text { if } & t=-\gamma \beta^{p}, \\
0 & \text { if } & t>-\gamma \beta^{p} .
\end{array}\right.
\end{array}
$$


The next lemma will later be used for an application of Lebesgue's dominated convergence theorem.

Lemma 7.5. (i) For all $\varepsilon>0$ and all sufficiently large $N$ we have

$$
g_{N}(t) \leqq \exp (-N(t-\varepsilon))
$$

uniformly in $t$.

(ii) There exist $c_{1}, c_{2}>0$ such that for all sufficiently large $N$

$$
1-g_{N}(t) \leqq c_{1} \exp \left(c_{2}\left(t+\gamma \beta^{p}\right)\right)
$$

uniformly in $t$.

Proof. (i) We apply Jensen's inequality to (7.18), making use of the fact that the function $x \mapsto 1-\exp (-x)$ is concave and strictly increasing:

$$
\begin{aligned}
g_{N}(t) & \leqq 1-\exp \left[-\int \varrho(d x) \exp \left(N^{1 / p} \beta x-N\left(t+\gamma \beta^{p}\right)\right)\right] \\
& \leqq 1-\exp [-\exp (-N(t-\varepsilon))] \\
& \leqq \exp (-N(t-\varepsilon)),
\end{aligned}
$$

where in the second inequality, we applied (2.2) in the form

$$
\int \varrho(d x) \exp \left(N^{1 / p} \beta x-N \gamma \beta^{p}\right) \leqq \exp (N \varepsilon)
$$

for all $\varepsilon>0$ and sufficiently large $N$.

(ii) It is possible to show that the property (2.1) of $\varrho$ implies that there exist positive numbers $\bar{c}_{1}, \bar{c}_{2}>0$ such that

$$
\varrho(-\infty, x] \leqq \bar{c}_{1} \exp \left(\bar{c}_{2} x\right)
$$

and

$$
\varrho[x,+\infty) \leqq \bar{c}_{1} \exp \left(-\bar{c}_{2} x\right)
$$

for all $x$. (The reader might also take this as additional assumptions on $\varrho$.)

Now we apply the inequality $\exp (-\exp (x)) \leqq \exp \left(-x^{+}\right)$where $x^{+}=\max (x, 0)$, to (7.17). We introduce the abbreviations

$$
\begin{aligned}
z & =N^{1 / q}\left(t+\gamma \beta^{p}\right) / \beta(\text { or } z=t / \beta-\gamma \text { if } p=1) \text { and } y=N^{1 / p} \beta(x-z) \\
1-g_{N}(t) & \leqq \int \varrho(d x) \exp \left(-N^{1 / p} \beta(x-z)^{+}\right) \\
& =\varrho(-\infty, z)+\int_{z}^{\infty} \varrho(d x) \exp \left(-N^{1 / p} \beta(x-z)\right) \\
& =\varrho(-\infty, z)+\int_{0}^{\infty} d y e^{-y} \varrho\left[z, z+y / N^{1 / p} \beta\right) \\
& =\int_{0}^{\infty} d y e^{-y} \varrho\left(-\infty, z+y / N^{1 / p} \beta\right) \\
& \leqq \bar{c}_{1} \int_{0}^{\infty} d y \exp \left(-y\left(1-\bar{c}_{2} / N^{1 / p} \beta\right)\right) \cdot \exp \left(\bar{c}_{2} z\right) \\
& \leqq c_{1} \exp \left(\bar{c}_{2} z\right)
\end{aligned}
$$




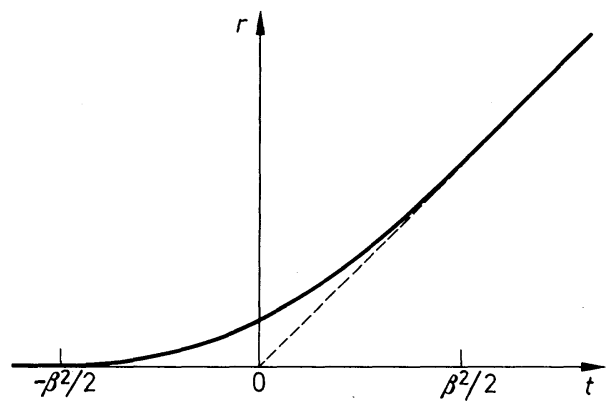

Fig. 6

with a new constant $c_{1}>\bar{c}_{1}>0$ and for $N$ sufficiently large. If $p=1$, then (7.27) is equivalent to (7.23). Since $1-g_{N}(t) \leqq 1$ for all $N$ and $t,(7.27)$ is only interesting for $z<0$, i.e. for $t+\gamma \beta^{p}<0$. But then, in the case $p>1$,

$$
z=N^{1 / q}\left(t+\gamma \beta^{p}\right) / \beta<\left(t+\gamma \beta^{p}\right) / \beta<0,
$$

and (7.23) follows again.

Now we shall study the limiting behaviour of $g_{N}(t)$ for $N \rightarrow \infty$ more precisely. This will turn out to be the essential mathematical point, which also produces the phase transition. While $g_{N}(t)$ converges to zero for $t>-\gamma \beta^{p}$ as stated in (7.21), even exponentially, the logarithmic rate of convergence depends in a peculiar way on $t$. To show this, we define for measures $\varrho$, satisfying the hypothesis (A) with $p>1$, $\gamma>0$

$$
r(t)=r(t, \beta, \varrho)= \begin{cases}0 & \text { for } t \leqq-\gamma \beta^{p} \\ \gamma(p-1)\left(t / p \gamma \beta+\beta^{p-1} / p\right)^{q} & \text { for }-\gamma \beta^{p} \leqq t \leqq(p-1) \gamma \beta^{p},(7.28) \\ t & \text { for }(p-1) \gamma \beta^{p} \leqq t\end{cases}
$$

while for a measure $\varrho$ with $p=1$, we put

$$
r(t)=r(t, \beta, \varrho)=\left\{\begin{array}{lll}
0 & \text { for } t \leqq 0 \\
t & \text { for } t \geqq 0
\end{array}\right.
$$

In (7.29) $r$ does not depend on $\beta$.

Example. If $\varrho$ is the standard Gaussian measure

$$
\varrho(d x)=\exp \left(-x^{2} / 2\right) / \sqrt{2 \pi} d x
$$

then $p=q=2, \gamma=\frac{1}{2}$, and

$$
r(t, \beta, \varrho)= \begin{cases}0 & t \leqq-\beta^{2} / 2 \\ (t / \beta+\beta / 2)^{2} / 2 & -\beta^{2} / 2 \leqq t \leqq \beta^{2} / 2 \\ t & \beta^{2} / 2 \leqq t\end{cases}
$$

(see Fig. 6). 


\section{Theorem 7.6.}

$$
\lim _{N \rightarrow \infty} N^{-1} \ln g_{N}(t)=-r(t, \beta, \varrho) .
$$

Proof. We first consider the case $p>1$. Substituting $u=\exp \left(N\left(z-t-\gamma \beta^{p}\right)\right)$ in (7.15), we obtain

$$
g_{N}(t)=N \int_{-\infty}^{+\infty} d z \exp \left\{N\left(z-t-\gamma \beta^{p}\right)-\exp \left(N\left(z-t-\gamma \beta^{p}\right)\right)+\ln \varrho\left[N^{1 / q} z / \beta, \infty\right)\right\} .
$$

Hence by (2.22) and arguments similar to (12.10)-(12.17), we get

$$
\begin{aligned}
\lim _{N} N^{-1} & \ln g_{N}(t) \\
& =\sup _{z}\left\{z-t-\gamma \beta^{p}+\lim _{t \rightarrow \infty}\left[\frac{-1}{r} \exp \left(r\left(z-t-\gamma \beta^{p}\right)\right)+\frac{1}{r} \ln \varrho\left[r^{1 / q} z / \beta, \infty\right)\right]\right\} \\
& =\sup _{z \leqq t+\gamma \beta^{p}}\left\{z-t-\gamma \beta^{p}-b(z / \beta)^{q} \cdot 1_{(0, \infty)}(z)\right\} .
\end{aligned}
$$

For $t+\gamma \beta^{p} \leqq 0$, the last expression is zero. Let $t+\gamma \beta^{p}>0$. The maximum of the function

$$
h(z)=z-t-\gamma \beta^{p}-b(z / \beta)^{q}
$$

is attained at

$$
z^{*}=(q b)^{-(p-1)} \beta^{p}=p \gamma \beta^{p}
$$

by (2.23). Thus

$$
\text { (7.34) }= \begin{cases}0 & \text { if } t+\gamma \beta^{p}<0, \\ h\left(t+\gamma \beta^{p}\right) & \text { if } 0 \leqq t+\gamma \beta^{p} \leqq p \gamma \beta^{p}, \\ h\left(p \gamma \beta^{p}\right) & \text { if } \quad p \gamma \beta^{p} \leqq t+\gamma \beta^{p} .\end{cases}
$$

It is now easy to show that

$$
(7.37)=-r(t, \beta, \varrho) .
$$

Assume now that $p=1$, i.e., by Proposition 2.1, that $\varrho$ has bounded support on $(0, \infty)$ and

Then

$$
\gamma=\operatorname{ess} \sup \varrho \uparrow(0, \infty) \text {. }
$$

$$
\begin{aligned}
g_{N}(t) & =\int_{0}^{\exp (-N t)} d u e^{-u} \varrho[\ln u / \beta N+t / \beta+\gamma, \infty) \\
& =N \int_{t}^{\infty} d z \exp \left\{N\left(-z-\exp (-N z) / N+\frac{1}{N} \ln \varrho\left[\frac{1}{\beta}(-z+t+\gamma \beta), \infty\right)\right)\right\},
\end{aligned}
$$

such that

$$
\lim N^{-1} \ln g_{N}(t)=\sup _{\substack{t \leqq z \\ 0 \leqq z}}\{-z\}=-r(t) .
$$

Thus (7.32) is proven for all cases.

Remark. We notice that the important non-analyticity of $\lim N^{-1} \ln g_{N}(t)$ at $t=(p-1) \gamma \beta^{p}$ is caused by the condition that the supremum in (7.34) is taken only 
over $z \leqq t+\gamma \beta^{p}$. To see this phenomenon in more detail, let $t>0$. Now for small values of $\beta$, we see from (7.36) that $z^{*}<t+\gamma \beta^{p}$. But when $\beta$ increases, then at $\beta=(t /(p-1) \gamma)^{1 / p}, z^{*}$ ceases to satisfy the condition $z \leqq t+\gamma \beta^{p}$. The supremum of (7.34) is attained at $z_{0}=t+\gamma \beta^{p}<z^{*}$.

A similar phenomenon occurs in the spherical model of T.H. Berlin and M. Kac. There the "sticking" of the saddle point in the three dimensional spherical model is responsible for the phase transition (see [1, footnote 8, p. 835]). As a mathematical mechanism for a phase transition, this "sticking" of the maximal point is completely different from a bifurcation phenomenon, which usually occurs in the phase transition of a mean field model.

In the next section we need the following consequence of Theorem 7.6:

Corollary 7.7. (I) Let $p>1$. Then

$$
\begin{aligned}
\lim _{N} \ln \left(-\ln \left(1-g_{N}(t)\right)\right) & =+\infty & \text { for } & t<-\gamma \beta^{p}, \\
\lim _{N} N^{-1} \ln \left(-\ln \left(1-g_{N}(t)\right)\right) & =-r(t, \beta, \varrho) & \text { for } & t \geqq-\gamma \beta^{p} .
\end{aligned}
$$

(II) For $p=1$, we have

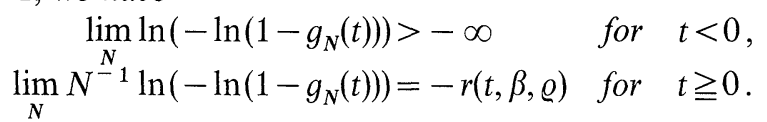

Proof. (i) and (iii): (7.41) is an immediate consequence of (7.21), and (7.43) is a consequence of (7.20).

We prove now (ii) and (iv) simultaneously, setting $t_{0}=-\gamma \beta^{p}$ if $p>1$ and $t_{0}=0$ if $p=1$. Assume first that $t>t_{0}$, and choose $\delta>0$ such that $0<\delta<r(t)-\delta$, which is possible since $r(t)>0$, for $t>t_{0}$. Now take $N_{0}$ so large that

$$
N_{0} \geqq \ln 2 \cdot(r(t)-\delta)^{-1} \geqq \ln 2 / \delta,
$$

and, by Theorem 7.7, such that

$$
-r(t)+\delta \geqq N^{-1} \ln g_{N}(t) \geqq-r(t)-\delta
$$

for all $N \geqq N_{0}$. We now use the inequality

$$
x \leqq-\ln (1-x) \leqq 2 x \text { for } 0 \leqq x \leqq 1 / 2,
$$

and notice that $\exp (-N(r(t)-\delta)) \leqq 1 / 2$ by (7.45). Hence

$$
2 \exp (-N(r(t)-\delta)) \geqq-\ln \left(1-g_{N}(t)\right) \geqq \exp (-N(r(t)+\delta))
$$

for $N \geqq N_{0}$ or, since $\ln 2 / N \leqq \delta$,

$$
-r(t)+2 \delta \geqq N^{-1} \ln \left(-\ln \left(1-g_{N}(t)\right)\right) \geqq-r(t)-\delta .
$$

This shows (7.42) and also (7.44) for the case $t>t_{0}$, since $\delta>0$ can be made arbitrarily small. If $t=t_{0}$ and $p>1$, then (7.42) follows from (7.21) again, since $\varrho(0, \infty)>0$. Finally let $p=1$ and $t=0$. Since $g_{N}$ is decreasing, we have

$$
\lim N^{-1} \ln \left(-\ln \left(1-g_{N}(0)\right)\right) \geqq-\lim _{t \downarrow 0} r(t)=0 .
$$

Conversely, by $(7.20) g_{N}(0)<1-1 / 2 e$ for all large $N$. Hence

$$
\lim N^{-1} \ln \left(-\ln \left(1-g_{N}(0)\right)\right) \leqq \lim N^{-1} \ln \ln (2 e)=0 .
$$




\section{Proof of Theorem 3.1}

We define $s=s(r)$ as the inverse function of $r$ on $[0, \infty)$ : i.e. if $p>1$, then

$$
s(r)=s(r, \beta, \varrho)= \begin{cases}p \gamma \beta((q-1) r / \gamma)^{1 / q}-\gamma \beta^{p} & \text { for } 0 \leqq r \leqq(p-1) \gamma \beta^{p}, \\ r & \text { for }(p-1) \gamma \beta^{p} \leqq r,\end{cases}
$$

while, for $p=1$, we have simply

$$
s(r)=r \text { for } r \geqq 0 .
$$

It is easily checked that $\psi$, as defined by (3.2), satisfies

$$
\psi(\beta, \varrho, \ln \kappa)=s(\ln \kappa, \beta, \varrho)-\ln \kappa .
$$

Note that if $p=1$, then $\psi \equiv 0$.

Equation (8.3) indicates a first connection between the random variable $\eta$ from (7.1) via (7.2), (7.16) and Theorem 7.6 to the right-hand side of Eq. (3.6) of Theorem 3.1. To establish a second connection between $\eta$ and $\ln Z_{N}$, we introduce the $\Phi$-function of $Z_{N}$ with the scaling factor $-\exp (-N t)$ :

$$
h_{N}(t)=\ln E \exp \left\{-\sum_{i=1}^{\kappa^{N}} \exp \left[N^{1 / p} \beta \zeta_{i}-N\left(t+\ln \kappa+\gamma \beta^{p}\right)\right]\right\} .
$$

Lemma 8.1.

$$
h_{N}(t)=-\exp \left\{N \ln \kappa+\ln \left(-\ln \left(1-g_{N}(t+\ln \kappa)\right)\right)\right\} \leqq 0 .
$$

Proof. With (7.2) and Lemma 7.3, we get

$$
\begin{aligned}
h_{N}(t) & =\kappa^{N} \varphi_{N}(-\exp (-N(t+\ln \kappa)))=-\kappa^{N}\left(-\ln \left(1-g_{N}(t+\ln \kappa)\right)\right) \\
& =-\exp \left\{N \ln \kappa+\ln \left(-\ln \left(1-g_{N}(t+\ln \kappa)\right)\right)\right\} . \quad \square
\end{aligned}
$$

\section{Lemma 8.2.}

$$
\lim _{N} h_{N}(t)= \begin{cases}-\infty & \text { for } t<\psi(\beta, \varrho, \ln \kappa), \\ 0 & \text { for } \quad t>\psi(\beta, \varrho, \ln \kappa) .\end{cases}
$$

Proof. We set again $t_{0}=-\gamma \beta^{p}$ if $p>1$ and $t_{0}>0$ if $p=1$. We remark that by the Definition (8.1), (8.2), and (8.3),

$$
t \lessgtr \psi(\beta, \varrho, \ln \kappa)=s(\ln \kappa, \beta, \varrho)-\ln \kappa
$$

is equivalent to

$$
r(t+\ln \kappa) \lessgtr \ln \kappa .
$$

Assume first that $t+\ln \kappa<t_{0}$, which implies $r(t+\ln \kappa)=0<\ln \kappa$. By (7.41) and (7.34), we have

$$
\ln \left(-\ln \left(1-g_{N}(t)\right) \geqq C>-\infty\right.
$$

for all $N$ and a suitable constant $C$. Lemma 8.1 implies

$$
h_{N}(t) \leqq-\exp (N \ln \kappa+C) \rightarrow-\infty \text { for } N \rightarrow \infty .
$$

Now take $t_{0} \leqq t+\ln \kappa$ but $r(t+\ln \kappa)<\ln \kappa$. Then by (7.42) and (7.44), there exists $\delta>0$ such that for all sufficiently large $N$

$$
\ln \kappa+N^{-1} \ln \left(-\ln \left(1-g_{N}(t+\ln \kappa)\right)\right)>\delta>0 .
$$


Hence by $(8.5) h_{N}(t) \leqq-\exp \{N \delta\} \rightarrow-\infty$. If $r(t+\ln \kappa)>\ln \kappa$, which means in particular $t+\ln \kappa>t_{0}$, then for all $N$ sufficiently large

$$
\ln \kappa+N^{-1} \ln \left(-\ln \left(1-g_{N}(t+\ln \kappa)\right)\right)<-\delta<0
$$

for some $\delta>0$. And thus

$$
0 \geqq h_{N}(t) \geqq-\exp (-N \delta) \rightarrow 0 \text { for } \quad N \rightarrow \infty .
$$

This shows (8.6).

To reverse the step from $N^{-1} \ln Z_{N}$ to $Z_{N}$, we make use of the following version of Frullani's integral formula (see [5, Sect. 495]):

$$
\begin{aligned}
N^{-1} \ln x= & \int_{-\infty}^{+\infty}\{\exp (-\exp (-N t))-\exp (-x \exp (-N t))\} d t \\
= & \int_{-\infty}^{y} \exp (-\exp (-N t)) d t-\int_{-\infty}^{y} \exp (-x \exp (-N t)) d t \\
& -\int_{y}^{\infty}\{1-\exp (-\exp (-N t))\} d t+\int_{y}^{\infty}\{1-\exp (-x \exp (-N t))\} d t,
\end{aligned}
$$

where $y \in \mathbb{R}$ is arbitrary. We take $y=\psi(\beta, \varrho, \ln \kappa) \leqq 0$, and we can now write in the same way as (8.8),

$$
N^{-1} E \ln Z_{N}=I_{1}+I_{2}+I_{3}+I_{4},
$$

with the following four integral expressions:

$$
\begin{aligned}
& I_{1}=\int_{-\infty}^{\psi} \exp (-\exp (-N t)) d t, \\
& I_{2}=-\int_{\psi}^{\infty}\{1-\exp (-\exp (-N t))\} d t, \\
& I_{3}=-\int_{-\infty}^{\psi} E \exp \left(-Z_{N} \exp (-N t)\right) d t, \\
& I_{4}=\int_{\psi}^{\infty}\left\{1-E \exp \left(-Z_{N} \exp (-N t)\right)\right\} d t .
\end{aligned}
$$

We can directly apply Lebesgue's dominated convergence theorem to $I_{1}$ and $I_{2}$, and we get

$$
\lim _{N \rightarrow \infty} I_{1}=0 \text { and } \quad \lim _{N \rightarrow \infty} I_{2}=\psi .
$$

Furthermore, we have by (8.4)

$$
I_{3}=-\int_{-\infty}^{\psi} \exp \left(h_{N}(t)\right) d t \quad \text { and } \quad I_{4}=\int_{\psi}^{\infty}\left\{1-\exp \left(h_{N}(t)\right)\right\} d t .
$$

Assume for a moment that we are allowed to apply Lebesgue's theorem to $I_{3}$ and $I_{4}$ also. Then Lemma 8.2 implies

$$
\lim _{N \rightarrow \infty} I_{3}=0 \text { and } \lim _{N \rightarrow \infty} I_{4}=0 .
$$


Equations (8.11) and (8.13) together give the desired result

$$
\lim _{N \rightarrow \infty} N^{-1} E \ln Z_{N}=\psi \text {. }
$$

We now justify the application of Lebesgue's theorem to $I_{3}$ and $I_{4}$ by using Lemma 7.5. For $I_{3}$, we take advantage of part (ii) of Lemma 7.5 and find an integer $N_{0}$ and positive constants $c_{1}, c_{2}>0$, where we can a fortiori assume that $c_{1} \geqq 1$, such that (7.23) holds for all $N \geqq N_{0}$ and all $t$. We define

$$
f_{1}(t)=\min \left\{1_{(-\infty, \psi]}(t), c_{1} \cdot \exp \left(c_{2}\left(t+\ln \kappa+\gamma \beta^{p}\right)\right)\right\},
$$

which is clearly integrable with respect to Lebesgue measure, and we shall show that for all $t$

$$
0 \leqq 1_{(-\infty, \psi]}(t) \cdot \exp \left(h_{N}(t)\right) \leqq f_{1}(t)
$$

or equivalently, that

$$
\exp \left(h_{N}(t)\right) \leqq c_{1} \exp \left(c_{2}\left(t+\ln \kappa+\gamma \beta^{p}\right)\right)
$$

With (8.5) and (7.23), we have

$$
\begin{aligned}
\exp \left(h_{N}(t)\right) & =\exp \left(\exp (N \ln \kappa) \ln \left(1-g_{N}(t+\ln \kappa)\right)\right) \\
& \leqq 1-g_{N}(t+\ln \kappa) \\
& \leqq c_{1} \cdot \exp \left[c_{2}\left(t+\ln \kappa+\gamma \beta^{p}\right)\right]
\end{aligned}
$$

This shows (8.17) as desired.

For $I_{4}$, we choose a fixed $\varepsilon>0$ and define the Lebesgue-integrable function

$$
f_{2}(t)=\min \left\{1_{[\psi, \infty)}(t), \exp (-(t-\varepsilon-\ln 2)\} .\right.
$$

By Lemma 7.5(i) we have for all sufficiently large $N$

$$
0 \leqq g_{N}(t+\ln \kappa) \leqq \exp (-N(t-\varepsilon+\ln \kappa))
$$

for all $t$. It suffices to show that (7.21) implies

$$
0 \leqq 1_{[\psi, \infty)}(t)\left(1-\exp \left(h_{N}(t)\right)\right) \leqq f_{2}(t)
$$

The last inequality is equivalent to

$$
1-\exp \left(h_{N}(t)\right) \leqq \exp (-(t-\varepsilon-\ln 2))
$$

for all $t$ with $t-\varepsilon-\ln 2 \geqq 0$. But if $t-\varepsilon-\ln 2 \geqq 0$ then also

$$
t-\varepsilon+\ln \kappa \geqq \ln 2 \text {. }
$$

Now suppose (8.20) and (8.23). Both imply

$$
0 \leqq g_{N}(t+\ln \kappa) \leqq \exp (-N(t-\varepsilon+\ln \kappa)) \leqq \exp (-N \ln 2) \leqq 1 / 2,
$$

such that we can apply the inequality (7.47) to $x=g_{N}(t+\ln \kappa)$. By this and (8.5), we get 


$$
\begin{aligned}
1-\exp \left(h_{N}(t)\right) & \leqq-h_{N}(t)=\left(-\ln \left(1-g_{N}(t+\ln \kappa)\right)\right) \cdot \exp (N \ln \kappa) \\
& \leqq 2 g_{N}(t+\ln \kappa) \exp (N \ln \kappa) \\
& \leqq 2 \exp (-N(t-\varepsilon)) \\
& \leqq \exp (-(t-\varepsilon-\ln 2)),
\end{aligned}
$$

which is just (8.22). This completes the proof of Theorem 3.1.

\section{Proof of Theorem 4.3}

First, we give another definition for the function $j$.

Lemma 9.1. For $q<0$ let $\tau(q)$ be the unique solution of

$$
\tau(q)+\ln \kappa-r(\tau(q)+\ln \kappa)-q=0 .
$$

Then

$$
j(q)=q-\tau(q)=\ln \kappa-r(\tau(q)+\ln \kappa) .
$$

Proof. If $p=1$, then $\tau(q)=q-\ln \kappa<0$, and (9.2) holds by definition (4.14). Thus, let $p>1$. The function $y-r(y)$ is strictly increasing on $\left(-\infty,(p-1) \gamma \beta^{p}\right]$ onto $(-\infty, 0]$. So (9.1) has a unique solution for $q<0$ with $\tau(q) \in\left(-\infty,(p-1) \gamma \beta^{p}\right]$. The second equality of (9.2) is just another formulation of (9.1). To show the first equality, set $\tilde{j}(q)=q-\tau(q)$. With $(9.1)$, we get

$$
\tilde{j}(q)-\ln \kappa=-r(q-\tilde{j}(q)+\ln \kappa)
$$

or, since $s$ is the inverse function of $r$, and $\psi(y)=s(y)-y$ by (8.3),

$$
q=s(\ln \kappa-\tilde{j}(q))-(\ln \kappa-\tilde{j}(q))=\psi(\ln \kappa-\tilde{j}(q)) .
$$

Hence, by the definition of $j(q)$ in (4.14), $\tilde{j}(q)=\ln \kappa-\psi^{-1}(q)=j(q)$.

To prove Theorem 4.3 , we set

$$
j_{N}(q)=N^{-1} \ln \left(-\ln \operatorname{Prob}\left\{N^{-1} Z_{N} \leqq q\right\}\right),
$$

and we have to show that for all $q<0$ and $\varepsilon>0$

$$
\lim _{N} \inf _{N}(q) \geqq j(q)-\varepsilon .
$$

Take $q^{\prime} \in(q, 0)$ such that

$$
j(q) \geqq j\left(q^{\prime}\right)>j(q)-\varepsilon / 2 .
$$

We set $\eta=\min \left(\varepsilon, q^{\prime}-q\right) / 2>0$, such that

$$
q-q^{\prime}+\eta<0, \quad \text { and } \quad j\left(q^{\prime}\right)-\eta>j(q)-\varepsilon .
$$

With $\tau^{\prime}=\tau\left(q^{\prime}\right)$ from (9.1), we get by (9.2)

$$
j\left(q^{\prime}\right)=q^{\prime}-\tau^{\prime}=\ln \kappa-r\left(\tau^{\prime}+\ln \kappa\right) .
$$

We now use the inequality

$$
\begin{aligned}
1_{(-\infty, q]}\left(N^{-1} \ln Z_{N}\right) & =1_{\left(-\infty, \exp \left(N\left(q-\tau^{\prime}\right)\right)\right]}\left(Z_{N} \exp \left(-N \tau^{\prime}\right)\right) \\
& \leqq \exp \left\{\exp \left(N\left(q-\tau^{\prime}\right)\right)-Z_{N} \exp \left(-N \tau^{\prime}\right)\right\}
\end{aligned}
$$


Hence, with the definition of $h_{N}\left(\tau^{\prime}\right)$ in (8.4)

$$
\ln \operatorname{Prob}\left\{N^{-1} \ln Z_{N} \leqq q\right\} \leqq \exp \left\{N\left(q-\tau^{\prime}\right)\right\}+h_{N}\left(\tau^{\prime}\right) .
$$

We replace $h_{N}\left(\tau^{\prime}\right)$ by $(8.5)$ and choose $N_{0}$ sufficiently large, such that by Corollary 7.7

$$
N^{-1} \ln \left(-\ln \left(1-g_{N}\left(\tau^{\prime}+\ln \kappa\right)\right)\right) \geqq-r\left(\tau^{\prime}+\ln \kappa, \beta, \varrho\right)-\eta
$$

for all $N \geqq N_{0}$. With (9.5), (9.9), we continue for such $N$

$$
\begin{aligned}
\exp \left(N j_{N}(q)\right) & \geqq-\exp \left\{N\left(q-\tau^{\prime}\right)\right\}+\exp \left\{N\left(\ln \kappa-r\left(\tau^{\prime}+\ln \kappa, \beta, \varrho\right)-\eta\right)\right\} \\
& =\exp \left\{N\left(j\left(q^{\prime}\right)-\eta\right)\right\}\left[1-\exp \left\{N\left(q-q^{\prime}+\eta\right)\right\}\right] .
\end{aligned}
$$

Using (9.8), we conclude for finally all $N$

$$
\exp \left(N j_{N}(q)\right) \geqq \exp \{N(j(q)-\varepsilon)\},
$$

which is equivalent to the desired inequality (9.6).

\section{Proof of Theorem 6.1}

It is useful to rewrite $\Phi_{N}$ in the following way:

$$
\Phi_{N}(t)=N^{-1} \ln E \exp \left(t \ln \kappa^{-N} \sum_{i=1}^{\kappa^{N}} \exp \left\{N \beta\left(\zeta_{i}-\gamma\right)\right\}\right) .
$$

We have to show that $\lim _{N} \Phi_{N}(t) \equiv 0$, if $p=1$. Recall that by $(2.5)$

$$
\gamma=\operatorname{ess} \sup \varrho>0 \text {. }
$$

We have to consider the following three cases:

(I) $\varrho=\delta_{\gamma}$,

(II) $\varrho\{\gamma\}>0$ and $\varrho(-\infty, \gamma)>0$;

(III) $\varrho(-\infty, \gamma)=1$.

Case (I) is the trivial one, because then $\zeta_{i}-\gamma=0$ almost surely and thus $\Phi_{N}(t) \equiv 0$ for all $N$ and all $t$. and

In the case (II), set

$$
\begin{gathered}
\eta=\varrho\{\gamma\} \in(0,1) \\
\varrho(A)=\varrho((A+\gamma) \cap(-\infty, \gamma)) / 1-\eta,
\end{gathered}
$$

the conditional measure of $\varrho$ with respect to the condition $(-\infty, \gamma)$. Let $\tilde{\zeta}_{i}$ be independent random variables, all distributed according to $\tilde{\varrho}$, and $\tilde{E}$ the corresponding expectation. Actually $\tilde{E}$ is the conditional expectation of the random variables $\zeta_{i}-\gamma$ under the condition $\left\{\zeta_{i}-\gamma<0\right.$ for $\left.i=1, \ldots, \kappa^{N}\right\}$. With this notation, we have

$$
\begin{aligned}
\Phi_{N}(t)= & N^{-1} \ln \left[\sum_{x}\left(\begin{array}{c}
\kappa^{N} \\
x \kappa^{N}
\end{array}\right) \eta^{x \kappa^{N}}(1-\eta)^{(1-x) \kappa^{N}}\right. \\
& \left.\cdot \tilde{E} \exp \left(t \ln \left\{x+\kappa^{-N} \sum_{j=1}^{(1-x) \kappa^{N}} \exp \left(N \beta \tilde{\zeta}_{i}\right)\right\}\right)\right],
\end{aligned}
$$


where in the summation $x$ runs through $\left\{0,1 / \kappa^{N}, 2 / \kappa^{N}, \ldots, 1\right\}$. Set

$$
x_{0}=\left[\eta \kappa^{N}\right] / \kappa^{N} \text {. }
$$

The square bracket $[z]$ denotes the greatest integer, majorized by $z$. We now drop the summation of $x$ in (10.5) and take only the term with $x_{0}$. To evaluate the logarithm of the binomial coefficients, we use Stirling's formula in the following version:

$$
\ln \left(\begin{array}{c}
k \\
\alpha k
\end{array}\right)=-k[\alpha \ln \alpha+(1-\alpha) \ln (1-\alpha)]-\frac{1}{2}(\ln k+\ln \alpha+\ln (1-\alpha)+\ln 2 \pi)+o(k) .
$$

We get

$$
\begin{aligned}
\Phi_{N}(t) \geqq & N^{-1} \frac{x_{0}\left(\ln \eta-\ln x_{0}\right)+\left(1-x_{0}\right)\left(\ln (1-\eta)-\ln \left(1-x_{0}\right)\right)}{1 / \kappa^{N}} \\
& -\frac{1}{2} \ln \kappa-\frac{1}{2 N}\left(\ln x_{0}+\ln \left(1-x_{0}\right)+\ln 2 \pi\right)+N^{-1} o\left(\kappa^{N}\right) \\
& +N^{-1} \ln \tilde{E} \exp \left(t \ln \left\{x_{0}+\kappa^{-N} \sum_{j=1}^{\left(1-x_{0}\right) \kappa^{N}} \exp (N \beta \tilde{\zeta})\right\}\right) .
\end{aligned}
$$

The function

$$
g(x)=x(\ln \eta-\ln x)+(1-x)(\ln (1-\eta)-\ln (1-x)),
$$

with $x \in[0,1]$, is strictly concave, non-positive with

$$
g(\eta)=g^{\prime}(\eta)=0, \quad g^{\prime \prime}(\eta)=\frac{-1}{\eta(1-\eta)}<0
$$

Since $\left|x_{0}-\eta\right| \leqq \kappa^{-N}$, the first term in the right hand side of (10.8) converges to zero. The same holds for the last term, since the expression

$$
x_{0}+\kappa^{-N} \sum_{j=1}^{\left(1-x_{0}\right) \kappa^{N}} \exp \left(N \beta \tilde{\zeta}_{j}\right)
$$

is bounded below by $\eta-\kappa^{-N}$ and above by 1 ; recall that $\tilde{\zeta}_{j}<0$ almost surely. Hence

$$
\lim _{N} \Phi_{N}(t) \geqq-\ln \kappa / 2 \quad \text { for all } t .
$$

We now prove that $\lim \Phi_{N}(t)$ is also bounded from above. Indeed, we find using (10.7) and (10.9)

$$
\begin{aligned}
\Phi_{N}(t)= & N^{-1} \ln \kappa^{-N} \sum_{x} \exp \left[\frac{g(x)}{1 / \kappa^{N}}+\frac{1}{2}(N \ln \kappa-\ln x-\ln (1-x)+\ln 2 \pi)+o\left(\kappa^{N}\right)\right. \\
& \left.+\ln \tilde{E} \exp \left(t \ln \left\{x+\kappa^{-N} \sum_{j=1}^{(1-x) \kappa^{N}} \exp \left(N \beta \tilde{\zeta}{ }_{j}\right)\right\}\right)\right] .
\end{aligned}
$$

Laplace's method now yields

$$
\lim \Phi_{N}(t) \leqq \ln \kappa / 2 \text { for all } t .
$$


But $\Phi=\lim \Phi_{N}$ is a convex function with

and, by (10.8) and (10.9).

$$
\Phi(0)=\lim \Phi_{N}(0)=0
$$

$$
-\ln \kappa / 2 \leqq \Phi(t) \leqq \ln \kappa / 2 \quad \text { for all } t
$$

Hence $\Phi \equiv 0$, which we wanted to show.

Finally we reduce case (III) to case (II). Since $\zeta_{i}-\gamma<0$ almost surely, we have for all $N$

$$
\Phi_{N}(t) \begin{cases}\leqq 0 & \text { for } t \geqq 0 \\ \geqq 0 & \text { for } t \leqq 0\end{cases}
$$

By (10.2), we can choose $\varepsilon>0$ arbitrarily small such that

$$
1>\eta=\varrho[\gamma-\varepsilon, \gamma]>0 \text {. }
$$

Define $\tilde{\zeta}_{i}=\max \left(\zeta_{i}, \tilde{\gamma}\right)$ with $\tilde{\gamma}=\gamma-\varepsilon$. The distribution $\tilde{\varrho}$ of $\tilde{\zeta}_{i}$ satisfies the condition of case (II) with $\gamma$ replaced by $\tilde{\gamma}$. In $(10.1)$, we go from $\Phi_{N}(t)$ to $\tilde{\Phi}_{N}(t)$ by replacing $\zeta_{i}$ and $\gamma$ by $\tilde{\zeta}_{i}$ and $\tilde{\gamma}$, respectively. Thus we get the following inequalities, where the upper $\geqq$ holds for $t \geqq 0$, while the lower $\leqq$ holds for $t \leqq 0$.

$$
\Phi_{N}(t) \leqq-\varepsilon \beta t+\tilde{\Phi}_{N}(t) .
$$

Since we know from case (II) $\lim _{N} \tilde{\Phi}_{N}(t)=0$, we pass to the limit $N \rightarrow \infty$ and then to $\varepsilon \downarrow 0$ in (10.17), thus getting

$$
\lim \Phi_{N}(t) \begin{cases}\geqq 0 & \text { for } t \geqq 0 \\ \leqq 0 & \text { for } t \leqq 0\end{cases}
$$

The results (10.15) and (10.18) together finish the proof in the case (III); and we are completely done.

\section{Proof of Theorem 6.2}

We keep the measure $\varrho$ with (2.2) and $p>1$ fixed. Trivially, we have $\Phi_{N}(0)=0$ for all $N$, hence $\Phi(0)=0$. By (2.2), we get

$$
\begin{aligned}
\Phi(1) & =\lim _{N} N^{-1} \ln E \exp \left(N^{1 / p} \beta \zeta-N \gamma \beta^{p}\right) \\
& =\beta^{p}\left[\lim _{N}\left(N^{1 / p} \beta\right)^{-p} \ln \int \exp \left(N^{1 / p} \beta x\right) \varrho(d x)-\gamma\right]=0 .
\end{aligned}
$$

Let now be $n \in[2,3,4, \ldots]$. Then

$$
\begin{aligned}
\Phi_{N}(n)= & N^{-1} \ln E\left(\sum_{i=1}^{\kappa^{N}} \exp \left\{N^{1 / p} \beta \zeta_{i}-N\left(\ln \kappa+\gamma \beta^{p}\right)\right\}\right)^{n} \\
= & N^{-1} \ln \sum_{\substack{l=1 \\
k_{1}, \ldots, k_{l} \geqq 1 \\
\sum k_{j}=n}} \frac{n !}{k_{1} ! \ldots k_{l} !} \kappa^{-N} \\
& \cdot \sum_{\substack{1 \leqq i_{j} \leqq \kappa^{N}, j=1, \ldots ., l \\
i ; \neq i, \text { for } i \neq i^{\prime}}} \cdot \prod_{j=1}^{l} E \exp \left(N^{1 / p} \beta k_{j} \zeta_{i_{j}}-N k_{j} \gamma \beta^{p}\right) .
\end{aligned}
$$


Hence

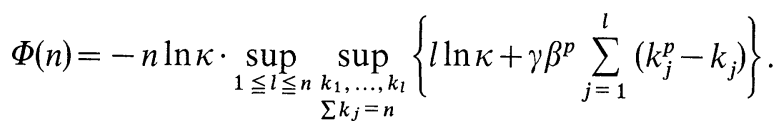

The function $\left(y_{1}, \ldots, y_{l}\right) \rightarrow \sum_{j=1}^{l}\left(y_{j}^{p}-y\right)$ is convex, and on the simplex $\left\{\left(y_{1}, \ldots, y_{l}\right), 1 \leqq y_{j}, \sum_{j=1}^{l} y_{j}=n\right\}$, it attains its maximum in a corner of the simplex. But the values of the function in the corners are all equal to $(n-l+1)^{p}-(n-l+1)$. Now the function

$$
y \rightarrow y \ln \kappa+\gamma \beta^{p}\left[(n-y+1)^{p}-(n-y+1)\right]
$$

is convex on $[0, n+1] \geqq[1, n]$. Thus, we get from (11.3) with the definition of $t(\beta)$ and $\hat{\Phi}$ in (6.8) and (6.9)

$$
\begin{aligned}
\Phi(n) & =\max \left\{0, \gamma \beta^{p}\left(n^{p}-n\right)-\ln \kappa(n-1)\right\} \\
& =\ln \kappa \cdot \max \left\{0,\left(n^{p}-n\right)\left[(q-1)\left(\beta / \beta_{C}\right)^{p}-\chi(n)\right]\right\} \\
& = \begin{cases}0 & \text { for } n<t(\beta), \\
\ln \kappa\left(n^{p}-n\right)\left[(q-1)\left(\beta / \beta_{C}\right)^{p}-\chi(n)\right] & \text { for } n \geqq t(\beta),\end{cases} \\
& =\hat{\Phi}(n) .
\end{aligned}
$$

So far, we have shown $\Phi(n)=\hat{\Phi}(n)$ for $n=0,1,2, \ldots$. By means of Theorem 3.1, a simple application of Jensen's inequality yields

$$
\begin{aligned}
\Phi(t) & =\lim N^{-1} \ln E \exp \left(t \cdot \ln Z_{N}\right) \\
& \geqq t \cdot \lim N^{-1} E \ln Z_{N}=t \cdot \psi(\beta, \varrho, \ln \kappa)
\end{aligned}
$$

for all $t \in \mathbb{R}$.

To prove the converse inequality for $t<0$, fix $t<0$ and let $\varepsilon$ with $0<\varepsilon<1$ be arbitrarily small. With $\psi=\psi(\beta, \varrho, \ln \kappa)$, we have now

$$
\begin{aligned}
\Phi_{N}(t) & \leqq N^{-1} \ln \sum_{k \in \mathbb{Z}} \exp (N t(\psi-\varepsilon+k)) \cdot \operatorname{Prob}\left\{\ln Z_{N} \in[N(\psi-\varepsilon+k), N(\psi-\varepsilon+k+1))\right\} \\
& =N^{-1} \ln \sum_{k} \exp \left[N\left(t(\psi-\varepsilon+k)-I_{N}([\psi-\varepsilon+k, \psi-\varepsilon+k+1))\right)\right]
\end{aligned}
$$

where

$$
I_{N}([\alpha, \beta))=-N^{-1} \ln \operatorname{Prob}\left\{N^{-1} \ln Z_{N} \in[\alpha, \beta)\right\} .
$$

By Theorem 4.1 we know that $I_{N}([\psi-\varepsilon+k, \psi-\varepsilon+k+1)) \rightarrow+\infty$ for $k \leqq-1$, while $I_{N}([\psi-\varepsilon, \psi-\varepsilon+1)) \rightarrow 0$. Thus, from (11.6) we conclude

$$
\Phi(t)=\lim _{N} \Phi_{N}(t) \leqq t(\psi-\varepsilon)
$$

The limit $\varepsilon_{\triangleleft} 0$ yields together with (11.5)

$$
\Phi(t)=t \cdot \psi(\beta, \varrho, \ln \kappa)
$$

for $t \in(-\infty, 0]$. This completes the proof of Theorem 6.2. 


\section{Proof of Theorem 2.3}

Let $p>1$ and $q$ be dual to $p$ in the sense of $(2.10): 1 / p+1 / q=1$. We start with several definitions. For $b \in[0, \infty]$, let

$$
\gamma(b):=\left\{\begin{array}{lll}
+\infty & \text { for } b=0, \\
p^{-1}(q b)^{-(p-1)} & \text { for } b \in(0, \infty), \\
0 & \text { for } b=+\infty .
\end{array}\right.
$$

Obviously,

$$
\gamma(b) \in(0, \infty) \text { if and only if } b \in(0, \infty)
$$

With

$$
g(y, b):=y-b y^{q} \quad \text { for } \quad y \geqq 0,
$$

we get

$$
\sup _{y \geqq 0} g(y, b)=\left.\left(y_{0}-b y_{0}^{q}\right)\right|_{y_{0}=(q b)-(p-1)}=\gamma(b),
$$

and since $y_{0}=p \gamma(b)>\gamma(b) / 2$, even

$$
\sup _{y \geqq \gamma(b) / 2} g(y, b)=\gamma(b) .
$$

By partial integration, we have for $t>0$

$$
\int \exp (t x) \varrho(d x)=\int \exp \left(t^{p} y\right) \varrho\left(d\left(t^{p-1} y\right)\right)=t^{p} \int \exp \left\{t^{p} h(y, t)\right\} d y,
$$

where

$$
h(y, t):=y+t^{-p} \ln \varrho\left[t^{p-1} y, \infty\right)=y-y^{q} b\left(t y^{q-1}\right),
$$

with

$$
b(\tau):=-\tau^{-p} \ln \varrho\left[\tau^{p-1}, \infty\right) .
$$

For short, set

$$
H(t):=t^{-p} \ln \int \exp \left\{t^{p} h(y, t)\right\} d y .
$$

Thus, we have to show that

$$
\lim _{\tau \rightarrow \infty} b(\tau)=b \in(0, \infty)
$$

is equivalent to

$$
\lim _{t \rightarrow \infty} H(t)=\gamma(b) \in(0, \infty)
$$

First, assume (12.10). Then for any $\varepsilon \in(0, b)$, there exists $C>0$ such that

$$
b-\varepsilon \leqq b(\tau) \leqq b+\varepsilon
$$

for all $\tau \geqq C$. Now for all $y \geqq \gamma(b+\varepsilon) / 2$ and all $t \geqq C /(\gamma(b+\varepsilon) / 2)^{q-1}$, we have by $(12.7)$ and (12.3)

$$
g(y, b+\varepsilon) \leqq h(y, t) \leqq g(y, b-\varepsilon) .
$$


Hence for all such $t$

$$
\begin{aligned}
t^{-p} \ln \int_{\gamma(b+\varepsilon) / 2}^{\infty} \exp \left\{t^{p} g(y, b+\varepsilon)\right\} d y & \leqq t^{-p} \ln \int_{\gamma(b+\varepsilon) / 2}^{\infty} \exp \left\{t^{p} h(y, t)\right\} d y \\
& \leqq t^{-p} \ln \int_{\gamma(b+\varepsilon) / 2}^{\infty} \exp \left\{t^{p} g(y, b-\varepsilon)\right\} d y
\end{aligned}
$$

By Laplace's method (cf. E. T. Copson: Asymptotic Expansions, Cambr. Univ. Press, 1971; Chap. 5) the left-, respectively, the right-hand side of (12.14) converges for $t \rightarrow \infty$ to

$$
\max _{y \geqq \gamma(b+\varepsilon) / 2} g(y, b+\varepsilon)=\gamma(b+\varepsilon)
$$

by (12.5), respectively, to

$$
\max _{y \geqq \gamma(b+\varepsilon) / 2} g(y, b-\varepsilon)=\gamma(b-\varepsilon) .
$$

For $y \leqq \gamma(b+\varepsilon) / 2$, we have for all $t$

$$
h(y, t) \leqq y \leqq \gamma(b+\varepsilon) / 2<\gamma(b+\varepsilon),
$$

such that we can conclude

$$
\gamma(b+\varepsilon)-\varepsilon \leqq H(t) \leqq \gamma(b-\varepsilon)+\varepsilon,
$$

for all $t$ sufficiently large. With $\varepsilon \rightarrow 0$, we finally get (12.11).

For the converse direction, we first show that

$$
\lim _{\tau \rightarrow \infty} b(\tau)=+\infty
$$

implies

$$
\limsup _{t \rightarrow \infty} H(t) \leqq 0 .
$$

Let $\varepsilon>0$, and $b>0$ such that $\gamma(b) \leqq \varepsilon$. By $(12.18) b(\tau) \geqq b$ for all large $\tau$. By (12.7), we get for all $y \geqq \varepsilon$ and all $t$ sufficiently large

$$
h(y, t) \leqq g(y, b) \leqq \gamma(b) \leqq \varepsilon .
$$

Since $h(y, t) \leqq y$ for all $y \geqq \varepsilon$, we get $\lim \sup H(t) \leqq \varepsilon$, which shows (12.19), since $\varepsilon>0$ can be made arbitrarily small.

Next, we prove that for $b \in(0, \infty)$,

$$
\liminf _{\tau \rightarrow \infty} \mathrm{b}(\tau) \leqq b
$$

implies

$$
\limsup _{t \rightarrow \infty} H(t) \geqq \gamma(b) .
$$

Let $\varepsilon>0$. From (12.21), we get a sequence $\left(\tau_{n}\right), \tau_{n} \rightarrow+\infty$, such that

$$
b\left(\tau_{n}\right) \leqq b+\varepsilon / 2
$$


for all $n$. Set $y_{0}=p \gamma(b+\varepsilon)$. Then by (12.4)

$$
g\left(y_{0}, b+\varepsilon / 2\right)>g\left(y_{0}, b+\varepsilon\right)=\gamma(b+\varepsilon) .
$$

Now, choose $\eta>0$ such that

$$
g\left(y_{0}+\eta, b+\varepsilon / 2\right)-2 \eta \geqq \gamma(b+\varepsilon) .
$$

In order to show (12.22), we take the sequence $\left(t_{n}\right), t_{n} \rightarrow \infty$, with

$$
t_{n}=\tau_{n}\left(y_{0}+\eta\right)^{-(q-1)}
$$

For all $y \in\left[y_{0}-\eta, y_{0}+\eta\right]$, we get

$$
\begin{aligned}
h\left(y, t_{n}\right) & \underset{(12.7)}{\gtrless}\left(y_{0}-\eta\right)+t_{n}^{-p} \ln \varrho\left[t_{n}^{p-1}\left(y_{0}+\eta\right), \infty\right) \\
& =\left(y_{0}+\eta\right)-\left(y_{0}+\eta\right)^{q} b\left(t_{n}\left(y_{0}+\eta\right)^{q-1}\right)-2 \eta \\
& \underset{(12.26)}{(12.23)}
\end{aligned}
$$

This implies limsup $H\left(t_{n}\right) \geqq \gamma(b+\varepsilon)$, and since $\varepsilon>0$ was arbitrary, we get (12.22) as desired.

Finally, we prove the following implication: If for $b \in(0, \infty)$

then

$$
\limsup _{\tau \rightarrow \infty} b(\tau)>b \geqq \liminf _{\tau \rightarrow \infty} b(\tau),
$$

$$
\liminf _{t \rightarrow \infty} H(t)<\gamma(b) \leqq \limsup _{t \rightarrow \infty} H(t) .
$$

The last inequality in (12.29) follows from (12.22). To show the first strict inequality, take $\varepsilon>0$ and a sequence $\left(\tau_{n}\right), \tau_{n} \rightarrow \infty$, with

$$
b\left(\tau_{n}\right) \geqq b+\varepsilon
$$

for all $n$. Since for $y_{0}=p \gamma(b)$,

$$
g\left(y_{0}, b+\varepsilon\right)<g\left(y_{0}, b\right)=\gamma(b)
$$

and

$$
g(y, b)<\gamma(b) \text { for all } y \neq y_{0},
$$

we find first $\eta, 0<\eta<\gamma(b) / 2 \leqq y_{0} / 2$, such that

$$
g\left(y_{0}-\eta, b+\varepsilon\right)+3 \eta \leqq \gamma(b),
$$

and then $\delta, 0<\delta<\min (b, \eta)$, with

$$
\max _{y \notin\left[y_{0}-\eta, y_{0}+\eta\right]} g(y, b-\delta)+\delta \leqq \gamma(b) .
$$

By (12.28), we have

$$
b(\tau) \geqq b-\delta \text { for all } \tau \geqq C,
$$

with a suitable constant $C>0$. We may assume that

$$
\tau_{n} \geqq C\left(\left(y_{0}-\eta\right) / \eta\right)^{q-1} .
$$


With

$$
t_{n}:=\tau_{n}\left(y_{0}-\eta\right)^{-(q-1)},
$$

we now prove that for all $y \in \mathbb{R}$ and all $n$

$$
h\left(y, t_{n}\right) \leqq K(y),
$$

where

$$
K(y)=\left\{\begin{array}{lll}
y & \text { for } y<\eta, \\
\gamma(b)-\eta & \text { for } y \in\left[y_{0}-\eta, y_{0}+\eta\right] \subseteq[\eta, \infty), \\
g(y, b-\delta) & \text { for } y \in[\eta, \infty)-\left[y_{0}-\eta, y_{0}+\eta\right] .
\end{array}\right.
$$

Remark that by (12.34),

$$
\sup _{y} K(y) \leqq \gamma(b)-\delta .
$$

For $y<\eta,(12.38)$ is evident from (12.7). For $y \in\left[y_{0}-\eta, y_{0}+\eta\right]$, we get

$$
\begin{aligned}
& h\left(y, t_{n}\right) \leqq\left(y_{0}+\eta\right)+t_{n}^{-p} \ln \varrho\left[t_{n}^{p-1}\left(y_{0}-\eta\right), \infty\right) \\
& \underset{(12.37)}{=}\left(y_{0}-\eta\right)-\left(y_{0}-\eta\right)^{q} b\left(\tau_{n}\right)+2 \eta \\
& \underset{(12.30)}{\leqq} g\left(y_{0}-\eta, b+\varepsilon\right)+2 \eta \underset{(12.33)}{\leqq} \gamma(b)-\eta=K(y) \text {. }
\end{aligned}
$$

Finally for $y \in[\eta, \infty)-\left[y_{0}-\eta, y_{0}+\eta\right],(12.36)$ and (12.37) yield

$$
t_{n} y^{q-1} \geqq C,
$$

and thus by (12.35)

$$
h\left(y, t_{n}\right)=y-y^{q} b\left(t_{n} y^{q-1}\right) \leqq g(y, b-\delta)=K(y) .
$$

This proves (12.38). But (12.38) and (12.40) imply

$$
\liminf _{t_{n} \rightarrow \infty} H\left(t_{n}\right) \leqq \liminf _{t} t^{-p} \ln \int \exp \left\{t^{p} K(y)\right\} d y \leqq \gamma(b)-\delta,
$$

which indeed proves the strict inequality in (12.29).

The implications (12.18-19), (12.21-22), and (12.28-29) together cover the converse direction of the equivalence (12.10) and (12.11). The proof of Theorem 2.3 is complete.

Acknowledgements. My thanks are due to J. L. van Hemmen for pointing out this problem to me and for valuable discussions.

\section{References}

1. Berlin, T.H., Kac, M.: The spherical model of a ferromagnet. Phys. Rev. 86, 821-835 (1952)

2. Derrida, B.: Random energy model: limit of a family of disordered models. Phys. Rev. Lett. 45, 79-82 (1980)

3. Dyson, F.J.: Existence and nature of phase transitions in one-dimensional Ising ferromagnets. In: Mathematical aspects of statistical mechanics SIAM-AMS proceedings. V. Providence, RI: Am. Math. Soc. 1972 
4. Ellis, R.S.: Large deviations and other limit theorems for a class of dependent random variables with applications to statistical mechanics. Preprint 1981

5. Fichtenholz, G.M.: Differential- und Integralrechnung. II. Berlin: Deutscher Verlag der Wissenschaften 1973

6. van Hemmen, J.L., Palmer, R.G.: The replica method and a solvable spin glass model. J. Phys. A 12, 563-580 (1979)

7. Lanford, O.E.: Entropy and equilibrium states in classical statistical mechanics. In: Statistical mechanics and mathematical problems, Battelle Seattle 1971. LN Phys. 20, 1-113 (1973)

8. Sherrington, D., Kirkpatrick, S. : Solvable model of a spin-glass. Phys. Rev. Lett. 35, 1792-1796 (1975)

Communicated by O. E. Lanford

Received January 3, 1983; in revised form March 25, 1983 
